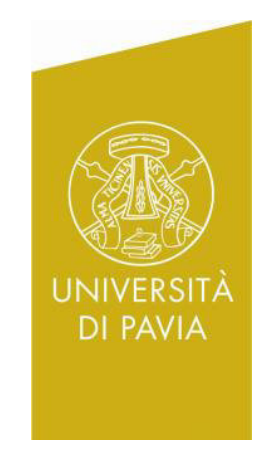

Department of Economics and Management DEM Working Paper Series

\title{
A General Theory of Endogenous Market Structures
}

\author{
Paolo Bertoletti \\ (Università di Pavia) \\ Federico Etro \\ (Università di Venezia, Ca' Foscari) \\ \# 81 (06-14) \\ Via San Felice, 5 \\ I-27100 Pavia \\ http://epmq.unipv.eu/site/home.html
}

June 2014 


\title{
A General Theory of Endogenous Market Structures
}

\author{
Paolo Bertoletti and Federico Etro ${ }^{1}$ \\ University of Pavia and Ca' Foscari University of Venice
}

First version: September 2013

This version: May 2014

Keywords: Monopolistic Competition, Imperfect Competition, Elasticity of Substitution, Free Entry

JEL Codes: D11, D43, L11

\begin{abstract}
We provide a unified approach to imperfect (monopolistic, Bertrand and Cournot) competition equilibria with demand functions derived from symmetric preferences over a large but finite number of goods. The equilibrium markups depend on the Morishima Elasticity of Substitution/Complementarity between goods, and can be derived directly from the utility functions and ranked unambiguously. We characterize the endogenous market structures, their dependence on market size, income and firms' productivity and compare them with the optimal allocations. Finally, we apply our results to the case of preferences such as Generalized Leontief, Generalized linear and Generalized quadratic that we introduce in the literature on imperfect competition.
\end{abstract}

\footnotetext{
${ }^{1}$ We are very grateful to Giuseppe Savaré for fundamental clarifications on the properties of symmetric functions, but of course the usual disclaimer applies. We also thank Paolo Epifani, Atsushi Kajii, Ina Simonovska and Kresimir Zigic for useful comments on an earlier version of this research. Seminar participants at the International Workshop on Global Competition and Innovation (Awaji, Japan, March 1, 2014), Kyoto University, Hitotsubashi University, Padova University and Yonsei University (Seoul) provided insightful comments. Correspondence. Paolo Bertoletti: Dept. of Economics and Management, University of Pavia, Via San Felice, 5, I-27100 Pavia, Italy. Tel: +390382986202, email: paolo.bertoletti@unipv.it. Federico Etro: Dept. of Economics, University of Venice Ca' Foscari, Sestiere Cannaregio, 30121, Fond.ta S.Giobbe 873, Venice, Italy. Tel: +390412349172, email: federico.etro@unive.it.
} 
Most of the modern theory of imperfect competition in markets with product differentiation is based on a few specific microfoundations of the demand side. Models of monopolistic competition are typically based on an additively separable direct utility as in Dixit and Stiglitz (1977) or on quasilinear preferences as in Spence (1976) and more recently Melitz and Ottaviano (2008). As well known, strategic interactions à la Cournot or Bertrand add a competitive element to the equilibrium of monopolistic competition, but their analysis too has been usually limited to few microfounded examples. The case of additively separable preferences have been recently reconsidered by Zhelobodko et al. (2012) and Bertoletti and Epifani (2014), and many results are known for the quasi-linear case: see Vives $(1999$, Ch. 6) for a comprehensive treatment. Other cases analyzed in the literature include those of homothetic preferences (Benassy, 1996, and Feenstra, 2003) and separable indirect utilities (Bertoletti and Etro, 2013). Beyond these cases, little is known about how general preferences shape competition and the incentives to enter/exit the market, that is the endogenous market structures. This is important not only for the partial equilibrium analysis of markets in industrial organization, but also for general equilibrium applications to trade and macroeconomics, most of which have been traditionally based on CES preferences. ${ }^{2}$

The purpose of this paper is to provide a unified approach to the analysis of imperfect competition, in its three main forms of monopolistic, Bertrand and Cournot competition, when consumers are endowed with general preferences that are symmetric over a large but finite number of differentiated goods. We first propose a generalized definition of monopolistic competition, and show that the alleged perceived demand elasticity coincides with the Morishima Elasticity of Substitution/Complementarity (as defined in Blackorby and Russell, 1981 and 1989), and can be derived directly from the utility function. We then show how the markups relevant in Bertrand and Cournot symmetric equilibria depend just on the same elasticities and on the number of varieties actually provided. On this basis, we characterize the implications of endogenous entry of firms and the comparative statics of the endogenous market structure in general. To illustrate our results we discuss the equilibria for a number of preferences, namely the case of separable direct or indirect utility, Translog preferences and quadratic direct utility, which have been already used in the literature on monopolistic or imperfect competition. Moreover, we present preferences that are so far unexplored in this literature, namely the Generalized Leontief preferences and the Generalized linear preferences from the homothetic class (see Diewert, 1971), and the Generalized quadratic preferences based on a quadratic indirect utility.

Our investigation allows us to shed some light on a number of key issues in the theory of imperfect competition. In the analysis of monopolistic competition we emphasize the role of the elasticity of substitution in shaping competition,

\footnotetext{
${ }^{2}$ There is a wide empirical literature pointing out that markups are affected by the number of consumers, their income and productivity shocks. For recent examples, see Campbell and Hopenhayn (2005) on competition effects in industrial organization, Simonovska (2013) on pricing to market in trade, and Nekarda and Ramey (2013) on the cyclicality of markups in macroeconomics.
} 
and we also provide a new example of (homothetic) preferences that deliver constant markups for any number of goods, and independently from income, market population and productivity (because the Morishima elasticity of substitution is constant as in the CES case). In the analysis of strategic interactions we show that Bertrand and Cournot markups are always higher than under monopolistic competition, and can be either decreasing or increasing in the number of firms competing in the market; as far as we know, we also provide the first computable example of Bertrand and Cournot equilibria with prices increasing in the number of competitors. As a consequence, endogenous market structures are characterized by a number of firms and an equilibrium markup that are increasing while moving from monopolistic competition to Bertrand and Cournot competition.

We fully characterize the comparative statics of the markups under endogenous market structures, stating the conditions on preferences under which there is neutrality or there are competitive effects with respect to changes in three key parameters: 1) the number of consumers, which represents market size in trade models or labor force in macroeconomics, 2) the individual income of consumers, whose changes correspond to cross-country income differences in trade and demand shocks in macroeconomics, and 3) the marginal cost, whose reduction corresponds to globalization in trade and a productivity boost in macroeconomics. The results are simple under monopolistic competition: the neutrality of population on markups holds not only with CES preferences (Krugman, 1980) but also under any additive indirect utility (Bertoletti and Etro, 2013); the neutrality of income holds under any additive direct utility (see Zhelobodko et al., 2012 and Bertoletti and Etro, 2014) and even with Generalized quadratic preferences; and the neutrality of the marginal cost holds under homothetic preferences, so that changes in productivity are translated proportionally on prices and do not affect the number of firms (see Bilbiie et al., 2012, for macroeconomic applications). Additional competitive effect emerge under Bertrand and Cournot competition. Finally, we characterize the optimal allocations and compare them with the endogenous market structures. For instance, excess entry occurs always with Translog, Generalized Leontief preferences and in the case of Generalized quadratic preferences. However, with Generalized linear preferences monopolistic competition generates insufficient (excess) entry if and only if markups are increasing (decreasing) in the number of firms, and efficiency when they are independent from the number of firms.

The paper is organized as follows: Section 1 describes the general model for a given number of firms and Section 2 illustrates it in examples, Section 3 endogenizes the number of firms, Section 4 discusses optimality and Section 5 concludes. Technical details are left to the Appendix.

\section{A General Model}

Consider a market populated by $L$ identical consumers with income $E>0$ to be spent on a number of differentiated goods, with price $p_{j}$ for variety $j$ 
whose individual consumption is given by $x_{j}$. We assume that the potential (arbitrarily large) number of varieties, $N$, is given, and that preferences over them are symmetric. However, only a number $n<N$ of varieties are actually provided in the market, and preferences can be represented equivalently by symmetric direct or indirect utility functions as:

$$
U^{n}=U^{n}(\mathbf{x}) \quad \text { or } \quad V^{n}=V^{n}(\mathbf{s}),
$$

where $s_{i}=p_{i} / E, i=1, \ldots n$ are the normalized prices. Notice that, as in an example by Feenstra (2003), utility functions (1) are naturally indexed by the number $n$, and can be endogenously derived from the underlying preferences (kept fixed) imposing that $N-n$ varieties have a price so high that their demands are nil. ${ }^{3}$ To satisfy standard conditions, it is assumed that $U^{n}(\cdot)$ is increasing and concave and that $V^{n}(\cdot)$ is decreasing and convex for any $n$.

The inverse and direct (Marshallian) demand systems are immediately provided by the following FOCs for utility maximization and Roy identities: ${ }^{4}$

$$
\mathbf{s}(\mathbf{x})=-\frac{D U^{n}(\mathbf{x})}{\mu^{n}(\mathbf{x})}, \quad \mathbf{x}(\mathbf{s})=\frac{D V^{n}(\mathbf{s})}{\mu^{n}(\mathbf{s})}
$$

where

$$
\mu^{n}(\mathbf{x})=-\sum_{j=1}^{n} U_{j}^{n}(\mathbf{x}) x_{j}=\sum_{j=1}^{n} V_{j}^{n}(\mathbf{s}) s_{j}=\mu^{n}(\mathbf{s}),
$$

is (minus) the marginal utility of income times $E$, and suffices denote partial derivatives. In Appendix A we show that symmetry of preferences implies that the derivatives:

$$
U_{i}^{n}(\mathbf{x})=h^{n}\left(x_{i}, \mathbf{x}\right) \text { and } V_{i}^{n}(\mathbf{s})=g^{n}\left(s_{i}, \mathbf{s}\right)
$$

are also symmetric with respect to $\mathbf{x}$ and $\mathbf{s}$, respectively for given $x_{i}$ and $s_{i}$. It follows that $\mu^{n}$ is symmetric too and that the inverse and direct demand functions:

$$
s_{i}\left(x_{i}, \mathbf{x}\right)=-\frac{h^{n}\left(x_{i}, \mathbf{x}\right)}{\mu^{n}(\mathbf{x})}, \quad x_{i}\left(s_{i}, \mathbf{s}\right)=\frac{g^{n}\left(s_{i}, \mathbf{s}\right)}{\mu^{n}(\mathbf{s})},
$$

$i=1, \ldots, n$, are symmetric with respect to their second (vector) arguments.

Suppose now that each variety is sold by a firm producing with constant marginal cost $c>0$ and fixed cost $F>0$. Accordingly, the profits of firm $i$ can be written as:

$$
\pi_{i}=\left(p_{i}-c\right) x_{i} L-F
$$

\footnotetext{
${ }^{3}$ Formally, let $U$ and $V$ to represent the preferences over the $N$ varieties, and suppose that $N-n$ of them are not available because the corresponding producers have not entered the market. Then $U^{n}(\mathbf{x})=U(\mathbf{x}, 0 \boldsymbol{\iota})$ and $V^{n}(\mathbf{s})=V\left(\mathbf{s}, s_{o}(\mathbf{s}) \boldsymbol{\iota}\right)$, where $\boldsymbol{\iota}$ is the unit vector of dimension $N-n$ and $s_{o}(\mathbf{s})$ is the choke-off price of the unavailable varieties, which possibly depends on the price of those actually provided. See the Appendix for examples of these derivations.

${ }^{4}$ Throughout this paper we assume that $\mathbf{x}(\mathbf{s})$ as well as $\mathbf{s}(\mathbf{x})$ behave well, and in particular are unique. For the sake of simplicity, we omit the index $n$.
} 
The equilibrium market structure depends on the form of competition, and we will examine (generalized) monopolistic competition, Bertrand competition and Cournot competition. First we will focus on equilibrium pricing and production decisions for a given number of varieties/firms, and then we will characterize the endogenous market structures.

\subsection{Generalized monopolistic competition}

According to (5), the direct impact of a firm choice on its demand is captured by the partial derivatives of demand with respect to its first argument:

$$
h_{1}^{n}\left(x_{i}, \mathbf{x}\right) \text { and } g_{1}^{n}\left(s_{i}, \mathbf{s}\right),
$$

while the indirect impact operates through the symmetric component of demand, and is analogous to the impact of competitors' choices. This suggests the following general definition of monopolistic competition, which extends the proposal by Dixit and Stiglitz (1977) of neglecting strategic interactions: ${ }^{5}$

Definition 1: Monopolistic competition characterizes a market structure in which each firm sets its choice ignoring the indirect impact on demand.

Notice that in many applications this is equivalent to say that each firm takes as given both the number of competitors $n$ and the value of some symmetric choice aggregators (this assumption would be trivially satisfied in any setting with a continuum of varieties). ${ }^{6}$ For example, this happens under direct additivity of preferences (Dixit and Stiglitz, 1977) or indirect additivity (Bertoletti and Etro, 2013), in which either $h^{n}$ or $g^{n}$ are constant with respect to the symmetric component, and monopolistic competition can be studied simply by taking the aggregator $\mu^{n}$ as given. Also notice that:

$$
x_{i}=h^{n-1}\left(-s_{i} \mu^{n}(\mathbf{x}(\mathbf{s})), \mathbf{x}(\mathbf{s})\right)=x_{i}\left(s_{i}, \mathbf{s}\right),
$$

that is, the partial inverse of $h^{n}$ with respect to its first argument gives the value of $x_{i}$, implying that the demand function perceived under monopolistic competition do not depend on the variable chosen to express the firm's choice.

According to our definition, the perceived inverse demand elasticity and the perceived direct demand elasticity in monopolistic competition are given by:

$$
\epsilon^{n}(\mathbf{x})=-\frac{h_{1}^{n}\left(x_{i}, \mathbf{x}\right) x_{i}}{h^{n}\left(x_{i}, \mathbf{x}\right)} \text { and } \varepsilon^{n}(\mathbf{s})=-\frac{s_{i} g_{1}^{n}\left(s_{i}, \mathbf{s}\right)}{g^{n}\left(s_{i}, \mathbf{s}\right)},
$$

which are of course one the reciprocal of the other when evaluated at $\mathbf{x}(\mathbf{s})$. Standard profit maximization shows that $\epsilon^{n}$ and $\varepsilon^{n}$ determine the optimal pricing rules under monopolistic competition:

\footnotetext{
${ }^{5}$ Notice that $h^{n}$ or $g^{n}$ might be independent respectively from $x_{i}$ and $s_{i}$ (given respectively $\mathbf{x}$ and $\mathbf{s})$. This is what happens in the case of homogeneous goods, where $h^{n}\left(\sum_{j=1}^{n} x_{j}\right)$ depends only on the total quantity consumed. Throughout this paper we assume on the contrary that $h^{n}$ and $g^{n}$ are monotonic with respect to their first arguments.

${ }^{6}$ This perspective suggests a possible way of generalizing our definition to the case of asymmetric preferences: see the cases of Generalized Additivity (Pollak, 1972) and Implicit Additivity (Hanoch, 1974).
} 
Proposition 1 In any equilibrium of Generalized monopolistic competition:

$$
\frac{p_{i}-c}{p_{i}}=\epsilon^{n}(\mathbf{x})=\frac{1}{\varepsilon^{n}(\mathbf{s})}
$$

Remarkably, the markup rules (8) would hold even in the case of marginal costs different across firms: accordingly, this analysis can be directly extended to the case of firm heterogeneity à la Melitz (2003). The explicit solution for the symmetric equilibrium price in which $\epsilon^{n}(x)=\epsilon^{n}(x \boldsymbol{\iota})$ and $\varepsilon^{n}(s)=\varepsilon^{n}(s \boldsymbol{\iota})$, where $\iota$ is the relevant unit vector, can be obtained using the budget constraint $n x s=1$. Notice that $(p-c) / p$ is a continuous, increasing function from $[c, \infty)$ onto $[0,1)$ : thus, a monopolistic competition equilibrium exists if $0<\epsilon^{n}(x)<1$ or $\varepsilon^{n}(s)>1 .^{7}$

\subsection{Perceived demand elasticity: a suggested interpreta- tion}

The interpretations of the monopolistic competition markup and the elasticities in (7) are not immediate. We provide an interpretation in terms of the (Morishima) Elasticity of Substitution, $\varepsilon_{i j}(\mathbf{s})$, and of the (Morishima) Elasticity of Complementarity, $\epsilon_{i j}(\mathbf{x})$, of the demand systems (2). These correspond to the elasticities of the price (quantity) ratios $s_{i} / s_{j}\left(x_{i} / x_{j}\right)$ with respect to the quantity (price) of good $i, j=1, \ldots, n, i \neq j$ (Blackorby and Russell, 1981):

$$
\epsilon_{i j} \equiv-\frac{\partial\left(s_{i} / s_{j}\right)}{\partial x_{i}} \frac{x_{i}}{\left(s_{i} / s_{j}\right)} \quad \text { and } \quad \varepsilon_{i j} \equiv-\frac{\partial\left(x_{i} / x_{j}\right)}{\partial s_{i}} \frac{s_{i}}{\left(x_{i} / x_{j}\right)},
$$

and can be directly computed from $s_{i} / s_{j}=U_{i}^{n}(\mathbf{x}) / U_{j}^{n}(\mathbf{x})$ and from $x_{i} / x_{j}=$ $V_{i}^{n}(\mathbf{s}) / V_{j}^{n}(\mathbf{s})$. These elasticities capture economically relevant aspects of the substitutability between goods (see Blackorby and Russell, 1989), which shapes the demand function of each firm. In our setting, the role of these measures emerges after rewriting the perceived demand elasticity. By symmetry of preferences, whenever $s_{i}=s_{j}$ and $x_{i}=x_{j}$, we have:

$$
h_{1}^{n}\left(x_{i}, \mathbf{x}\right)=U_{i i}^{n}(\mathbf{x})-U_{i j}^{n}(\mathbf{x}) \text { and } g_{1}^{n}\left(s_{i}, \mathbf{s}\right)=V_{i i}^{n}(\mathbf{s})-V_{i j}^{n}(\mathbf{s}),
$$

Therefore, it follows that:

$$
\epsilon^{n}=\frac{x_{i} U_{j i}^{n}(\mathbf{x})}{U_{j}^{n}(\mathbf{x})}-\frac{x_{i} U_{i i}^{n}(\mathbf{x})}{U_{i}^{n}(\mathbf{x})}=\epsilon_{i j}(\mathbf{x}) \text { and } \varepsilon^{n}=\frac{s_{i} V_{j i}^{n}(\mathbf{s})}{V_{j}^{n}(\mathbf{s})}-\frac{s_{i} V_{i i}^{n}(\mathbf{s})}{V_{i}^{n}(\mathbf{s})}=\varepsilon_{i j}(\mathbf{s}),
$$

where we used symmetry again to replace $U_{i}^{n}(\mathbf{x})$ with $U_{j}^{n}(\mathbf{x})$ and $V_{i}^{n}(\mathbf{s})$ with $V_{j}^{n}(\mathbf{s})$. Accordingly, the perceived demand elasticity that is relevant for firms active under monopolistic competition corresponds to the elasticity of the relative demand of two goods when their relative price changes (starting from identical prices).

\footnotetext{
${ }^{7}$ Sufficient conditions for uniqueness of the equilibrium are obvioulsy $\partial \varepsilon^{n}(s) / \partial s \geq 0$ and $\partial \epsilon^{n}(x) / \partial x \leq 0$ for any $x, s$.
} 
Our result generalizes the role of the constant elasticity of substitution under CES preferences in shaping monopolistic markups. It also provides a rationale for the well-known fact that, under direct additivity of preferences (Dixit and Stiglitz, 1977), the perceived demand elasticity only depends on the individual consumption of each variety and, in particular, on the (reciprocal of the) elasticity of its own marginal utility of consumption, which Zhelobodko et al. (2012) refer to as a "relative love for variety." The general result also explains why under indirect additivity (Bertoletti and Etro, 2013) the perceived demand elasticity of each firm depends only on the price-income ratio of its own good. In addition, the result that with homothetic preferences the symmetric demand elasticity only depends on $n$ (Benassy, 1996) follows from the homogeneity properties of the previous expressions under homotheticity. Of course, the formulas apply in the more general case of non-additive and non-homothetic preferences, opening up new scenarios for analysis. Our perspective will also illuminate the relationship between the elasticities perceived under monopolistic competition and the demand elasticities relevant under strategic interaction, as shown in next sections. ${ }^{8}$

\subsection{Cournot competition}

Consider a Cournotian equilibrium in our market:

Definition 2: Cournot competition characterizes a market structure in which each firm sets its production level taking in consideration its total impact on the inverse demand.

The relevant "individual marginal revenue" of firm $i, M R_{i}=\partial\left\{p_{i} x_{i}\right\} / \partial x_{i}$, can be written as:

$$
\begin{gathered}
M R_{i}=\frac{\left[U_{i}^{n}(\mathbf{x})+U_{i i}^{n}(\mathbf{x}) x_{i}\right] \mu^{n}(\mathbf{x})+U_{i}^{n}(\mathbf{x}) x_{i}\left[U_{i}^{n}(\mathbf{x})+\sum_{j=1}^{n} U_{j i}^{n}(\mathbf{x}) x_{j}\right]}{-\mu^{n}(\mathbf{x})^{2} / E} \\
=p_{i}(\mathbf{x})\left\{1-s_{i}(\mathbf{x}) x_{i}+\frac{U_{i i}^{n}(\mathbf{x}) x_{i}}{U_{i}^{n}(\mathbf{x})}-\sum_{j=1}^{n} \frac{U_{j i}^{n}(\mathbf{x}) x_{i}}{U_{j}^{n}(\mathbf{x})} s_{j}(\mathbf{x}) x_{j}\right\} \\
=p_{i}(\mathbf{x})\left\{1-s_{i}(\mathbf{x}) x_{i}-\sum_{j \neq i}^{n}\left[\frac{U_{j i}^{n}(\mathbf{x}) x_{i}}{U_{j}^{n}(\mathbf{x})}-\frac{U_{i i}^{n}(\mathbf{x}) x_{i}}{U_{i}^{n}(\mathbf{x})}\right] s_{j}(\mathbf{x}) x_{j}\right\} \\
=p_{i}(\mathbf{x})\left\{1-s_{i}(\mathbf{x}) x_{i}-\sum_{j \neq i}^{n} \epsilon_{i j}(\mathbf{x}) s_{j}(\mathbf{x}) x_{j}\right\} \\
=p_{i}(\mathbf{x}) \sum_{j \neq i}^{n}\left[1-\epsilon_{i j}(\mathbf{x})\right] s_{j}(\mathbf{x}) x_{j},
\end{gathered}
$$

${ }^{8} \mathrm{An}$ exploration of this relationship in the case of direct additivity of preferences is in Bertoletti and Epifani (2014). 
where $\epsilon_{i j}(\mathbf{x})$ is again the elasticity of complementarity between varieties $i$ and $j$. Notice that positive marginal revenues require that "on average" $\epsilon_{i j}(\mathbf{x})<1$. Accordingly, in a Cournot equilibrium we have:

$$
\frac{p_{i}(\mathbf{x})-c}{p_{i}(\mathbf{x})}=s_{i}(\mathbf{x}) x_{i}+\sum_{j \neq i}^{n} \epsilon_{i j}(\mathbf{x}) s_{j}(\mathbf{x}) x_{j}
$$

which provides a general markup rule.

Focusing on the case of a symmetric equilibrium, the markup becomes:

$$
\frac{p-c}{p}=\frac{1}{n}+\frac{n-1}{n} \epsilon^{n}(x),
$$

which exhibits a simple relationship with the pricing rule of the monopolistic competition equilibrium. Using $\epsilon^{n}(x)=1 / \varepsilon^{n}\left(s^{c}\right)$ we can express the same rule in terms of the elasticity of substitution drawn from the indirect utility. This proves the following result:

Proposition 2 In any symmetric Cournot equilibrium:

$$
\frac{p^{c}-c}{p^{c}}=\frac{1}{n}+\frac{n-1}{n} \epsilon^{n}\left(x^{c}\right)=\frac{1}{n}+\frac{n-1}{n \varepsilon^{n}\left(s^{c}\right)} .
$$

In Appendix B we also provide a constructive proof of the second equality appearing in (11). Notice that $1>\frac{1}{n}+\frac{n-1}{n} \epsilon^{n}(x)>\epsilon^{n}(x)$ if $\epsilon^{n}<1$. Accordingly, the assumption $\epsilon^{n}<1$ allows to rank the equilibrium markups in Cournot and monopolistic competition. This ranking depends on the fact that Cournotian firms internalize the indirect impact of their production level on marginal revenues, which is the same as the increase of production by any competitor: since goods are substitutes, this impact is negative and internalizing it amounts to reduce production and increase markups. More formally, let us differentiate $\sum_{j=1}^{n} s_{j}(\mathbf{x}) x_{j}=1$ with respect to $x_{i}$ making use of (5). In a symmetric equilibrium $(i \neq j)$ this gives:

$$
1=-\sum_{j=1}^{n} \frac{\partial s_{j}}{\partial x_{i}} \frac{x}{s}=\epsilon^{n}-n \frac{\partial s_{j}}{\partial x_{i}} \frac{x}{s} .
$$

$\epsilon^{n}<1$ implies that $\partial s_{j} / \partial x_{i}<0$, i.e., that varieties are gross, $q$-substitutes (see e.g. Bertoletti, 2005 on this terminology) in a symmetric equilibrium. Accordingly, Cournotian firms face an elasticity of the inverse demand $\epsilon^{n}-\frac{\partial s_{j}}{\partial x_{i}} \frac{x}{s}$ which is larger than the one perceived by firms in monopolistic competition, and actually price higher.

\subsection{Bertrand competition}

In a Bertrand version of our setting firms choose prices: 
Definition 3: Bertrand competition characterizes a market structure in which each firm sets its price taking in consideration its total impact on the direct demand.

Firms correctly perceive the true elasticity of Marshallian direct demands (5), that is:

$$
\begin{gathered}
\left|\frac{\partial x_{i}}{\partial p_{i}} \frac{p_{i}}{x_{i}}\right|=-\frac{s_{i}}{x_{i}(\mathbf{s})} \frac{V_{i i}^{n}(\mathbf{s}) \mu^{n}(\mathbf{s})-V_{i}^{n}(\mathbf{s})\left[V_{i}^{n}(\mathbf{s})+\sum_{j=1}^{n} V_{j i}^{n}(\mathbf{s}) s_{j}\right]}{\left[\mu^{n}(\mathbf{s})\right]^{2}} \\
=-\frac{s_{i} V_{i i}^{n}(\mathbf{s})}{V_{i}^{n}(\mathbf{s})}+\frac{s_{i}}{\mu^{n}(\mathbf{s})}\left[V_{i}^{n}(\mathbf{s})+V_{i i}^{n}(\mathbf{s}) s_{i}+\sum_{j \neq i}^{n} V_{j i}^{n}(\mathbf{s}) s_{j}\right] \\
=-\frac{s_{i} V_{i i}^{n}(\mathbf{s})}{V_{i}^{n}(\mathbf{s})}\left[1-s_{i} x_{i}(\mathbf{s})\right]+s_{i} x_{i}(\mathbf{s})+\sum_{j \neq i}^{n} \frac{s_{i} V_{j i}^{n}(\mathbf{s})}{V_{j}^{n}(\mathbf{s})} s_{j} x_{j}(\mathbf{s}) \\
=s_{i} x_{i}(\mathbf{s})+\sum_{j \neq i}^{n}\left[\frac{s_{i} V_{j i}^{n}(\mathbf{s})}{V_{j}^{n}(\mathbf{s})}-\frac{s_{i} V_{i i}^{n}(\mathbf{s})}{V_{i}^{n}(\mathbf{s})}\right] s_{j} x_{j}(\mathbf{s}) \\
=s_{i} x_{i}(\mathbf{s})+\sum_{j \neq i}^{n} \varepsilon_{i j}(\mathbf{s}) s_{j} x_{j}(\mathbf{s}) \\
=1+\sum_{j \neq i}^{n}\left[\varepsilon_{i j}(\mathbf{s})-1\right] s_{j} x_{j}(\mathbf{s}),
\end{gathered}
$$

where $\varepsilon_{i j}(\mathbf{s})$ is the elasticity of substitution between varieties $i$ and $j$. Notice that to satisfy the FOCs for profit maximization it is necessary that "on average" $\varepsilon_{i j}(\mathbf{s})>1$. In a symmetric Bertrand equilibrium:

$$
\frac{p-c}{p}=\frac{n}{1+(n-1) \varepsilon^{n}(s)} .
$$

Again using $\epsilon^{n}(x)=1 / \varepsilon^{n}\left(s^{c}\right)$, or inverting the inverse demand system (5) under symmetry (see Appendix B), we prove the following result:

Proposition 3 In any symmetric Bertrand equilibrium:

$$
\frac{p^{b}-c}{p^{b}}=\frac{n}{1+(n-1) \varepsilon^{n}\left(s^{b}\right)}=\frac{n \epsilon^{n}\left(x^{b}\right)}{\epsilon^{n}\left(x^{b}\right)+n-1} .
$$

Notice that $\left[1+(n-1) \varepsilon^{n}(s)\right] / n>\varepsilon^{n}(s)$ if $\varepsilon^{n}>1$. Thus, the assumption $\varepsilon^{n}>1$ allows us to rank the equilibrium markups in Bertrand and monopolistic competition. The intuition is again simple and relies on the internalization of the indirect impact of a price increase on demand under Bertrand competition. Differentiating $\sum_{j} s_{j} x_{j}(\mathbf{s})=1$ with respect to $s_{i}$ and making use of (5) yields that, in a symmetric equilibrium $(i \neq j)$ : 


$$
1=-\sum_{j=1}^{n} \frac{\partial x_{j}}{\partial s_{i}} \frac{s}{x}=\varepsilon^{n}-n \frac{\partial x_{j}}{\partial s_{i}} \frac{s}{x} .
$$

$\varepsilon^{n}>1$ implies that $\frac{\partial x_{j}}{\partial s_{i}}>0$, i.e. that varieties are gross, $p$-substitutes (again see e.g. Bertoletti, 2005). Accordingly, Bertrand firms perceive the true Marshallian elasticity $\epsilon^{n}-\frac{\partial x_{j}}{\partial s_{i}} \frac{s}{x}$ which is smaller than the one perceived by firms in monopolistic competition, and actually price higher.

Notice also that the Bertrand markup is always below the Cournotian one, in line with the general result due to Vives (1985). Therefore, we can summarize the ranking of markups as follows:

Corollary 4 Assume $\varepsilon^{n}>1\left(\epsilon^{n}<1\right)$ everywhere: then for any Cournot equilibrium markup there exists a lower Bertrand equilibrium markup which in turn is larger than a generalized monopolistic competition equilibrium markup.

\section{Old and New Examples}

In this section we review known particular cases of our general model, and propose some new applications. ${ }^{9}$

\subsection{Dixit-Stiglitz preferences}

Dixit and Stigliz (1977) adopted direct additivity, that is:

$$
U^{n}(\mathbf{x})=\sum_{j=1}^{n} u\left(x_{j}\right)
$$

with $u^{\prime \prime}(x)<0<u^{\prime}(x)$ for $x>0$. Such a functional form does not actually change with $n$ (provided that $u(0)=0$ ), and implies that $U_{j i}^{n}=0$ : the elasticity of complementarity is then $\epsilon_{i j}=-x_{i} u^{\prime \prime}\left(x_{i}\right) / u^{\prime}\left(x_{i}\right)=\epsilon\left(x_{i}\right)$, which does not depend on the number of competitors. The imperfect competition symmetric equilibria can be derived as:

$$
p=\frac{c}{1-\epsilon(x)}, \quad p^{b}=\frac{\left[\epsilon\left(x^{b}\right)+(n-1)\right] c}{(n-1)\left(1-\epsilon\left(x^{b}\right)\right)} \quad \text { and } \quad p^{c}=\frac{n c}{(n-1)\left(1-\epsilon\left(x^{c}\right)\right)} .
$$

The monopolistic competition markup can be decreasing or increasing in the number of firms, and in the latter case also the Cournot and Bertrand markups can be increasing for a large enough number of firms (see Bertoletti et al., 2008).

\footnotetext{
${ }^{9}$ Extending our definition of generalized monopolistic competition to settings with outside commodity (that is with indirect utility function as $\tilde{V}\left(s_{0}, V^{n}(\mathbf{s})\right)$, where $s_{0}$ is the priceincome ratio of the outside good) is straightforward: for an example with indirect additivity see Bertoletti and Etro (2013). On quasilinear preferences see Vives (1999) and Anderson et al. (2012).
} 


\subsection{Indirectly additive preferences}

Bertoletti and Etro (2013) have assumed indirect additivity, that is:

$$
V^{n}(\mathbf{s})=\sum_{j=1}^{n} v\left(s_{j}\right)
$$

with $v^{\prime \prime}(s)>0>v^{\prime}(s)$. Such a functional form again does not change with $n$ (if $v=0$ for the varieties that are not available) and implies $V_{j i}^{n}=0$ : the elasticity of substitution is $\varepsilon_{i j}=-s_{i} v^{\prime \prime}\left(s_{i}\right) / v^{\prime}\left(s_{i}\right)=\varepsilon\left(s_{i}\right)$, which again does not depend on the number of firms. The three symmetric equilibria can be derived as:

$$
p=\frac{\varepsilon(s) c}{\varepsilon(s)-1}, \quad p^{b}=\frac{\left[1+(n-1) \varepsilon\left(s^{b}\right)\right] c}{(n-1)\left(\varepsilon\left(s^{b}\right)-1\right)} \quad \text { and } \quad p^{c}=\frac{n \varepsilon\left(s^{c}\right) c}{(n-1)\left(\varepsilon\left(s^{c}\right)-1\right)} .
$$

Now markups are independent from the number of firms under monopolistic competition and decreasing with respect to $n$ under strategic interactions.

\subsection{Homothetic preferences}

Benassy (1996) discussed some instances of homothetic preferences: in that case, since $\epsilon_{i j}$ and $\varepsilon_{i j}$ are homogeneous of degree zero under homotheticity, the symmetric equilibrium markups must be a function of the number of varieties. ${ }^{10}$

\subsubsection{Translog preferences}

Feenstra (2003) has shown that symmetric Translog preferences imply:

$$
V^{n}(\mathbf{s})=\exp -a_{0}-\frac{1}{n} \sum_{j=1}^{n} \log s_{j}-\frac{1}{2} \sum_{k=1}^{n} \sum_{j=1}^{n} a_{k j} \log s_{k} \log s_{j}
$$

where $a_{0}=\alpha_{0}+1 /(2 \gamma n)-1 /(2 \gamma N), a_{k j}=\gamma / n>0$ for $k, j=1, \ldots, n, k \neq j$ and $a_{j j}=-\gamma(n-1) / n$, with $\gamma>0$. Applying the Roy identity we can express the individual demand of $\operatorname{good} i$ as:

$$
x_{i}=\left(\frac{1}{n p_{i}}-\gamma \frac{\log p_{i}}{p_{i}}+\frac{\gamma}{n} \sum_{j=1}^{n} \frac{\log p_{j}}{p_{i}}\right) E .
$$

In a symmetric equilibrium we have $\varepsilon^{n}=1+\gamma n$, and the three equilibria can be derived as follows:

$$
p=\left(1+\frac{1}{\gamma n}\right) c, \quad p^{b}=\left(1+\frac{1}{\gamma(n-1)}\right) c \quad \text { and } \quad p^{c}=\frac{1+\gamma n}{\gamma(n-1)} c .
$$

Markups are decreasing in the number of goods and would become negligible when $n \rightarrow N$ if the number of potential varieties $N$ is large.

\footnotetext{
${ }^{10}$ It is worth noticing that the classes of directly additive, indirectly additive or homothetic preferences have only a common member, given by the CES preferences.
} 


\subsubsection{Generalized Leontief preferences}

Another member of the class of so-called flexible functional forms (see Varian, 1992, Ch. 12.10) is given by the (homothetic) Generalized Leontief preferences, originally presented in Diewert (1971) and unexplored in the analysis of imperfect competition. Over a set of $N$ potential goods, these preferences can be represented by the following indirect utility function:

$$
V=\left[\sqrt{\mathbf{s}}^{\prime} \mathbf{B} \sqrt{\mathbf{s}}\right]^{-1}
$$

where $\sqrt{\mathbf{s}}$ is the vector of the square roots of the normalized prices, and $\mathbf{B}$ is a $N \times N$ matrix whose elements, to achieve symmetry (and to satisfy regularity assumptions), are $b_{k j}=b>0$ and $b_{j j}=\underline{b}<0$ for $k, j=1, \ldots, N, k \neq j$. It can be shown (see Appendix $C$ ) that, under the normalizations $b=1$ and $\underline{b}=-(N-2)$, we have:

$$
V^{n}=\left[\sum_{k=1}^{n} \sum_{j=1}^{n} a_{k j} \sqrt{s_{k} s_{j}}\right]^{-1}
$$

where

$$
a_{i i}=\underline{a}=\frac{-(N-1)(n-2)}{n-1}, \quad a_{i j}=a=\frac{N-1}{n-1} .
$$

Accordingly, we obtain the following demand for good $i$ :

$$
x_{i}=\frac{\underline{a}-a+a \sum_{k=1}^{n} \sqrt{\frac{s_{k}}{s_{i}}}}{\sum_{k=1}^{n} \sum_{j=1}^{n} a_{k j} \sqrt{s_{k} s_{j}}} .
$$

Since the perceived demand elasticity is:

$$
\varepsilon^{n}=\frac{\frac{a}{2} \sum_{k=1}^{n} \sqrt{\frac{s_{k}}{s_{i}}}}{\underline{a}-a+a \sum_{k=1}^{n} \sqrt{\frac{s_{k}}{s_{i}}}}
$$

in a symmetric equilibrium we have:

$$
\varepsilon^{n}=\frac{n a}{2(\underline{a}-a+n a)}=\frac{n}{2}
$$

This allows us to directly compute the three symmetric equilibria:

$$
p=\frac{n c}{n-2}, \quad p^{b}=\frac{\left(2+n^{2}-n\right) c}{2+n^{2}-3 n} \quad \text { and } \quad p^{c}=\frac{n^{2} c}{n^{2}-3 n+2} .
$$

Again, the markups decrease with the number of varieties and would become negligible when $n \rightarrow N$ if $N$ is large. 


\subsubsection{Generalized linear preferences}

We now consider another class of homothetic preferences that has not been exploited in the analysis of imperfect competition. This is represented by the following utility function (see Diewert, 1971, who associated it with Allen, McFadden and Samuelson):

$$
U=\sqrt{\mathbf{x}}^{\prime} \mathbf{B} \sqrt{\mathbf{x}}
$$

where the elements of the $N \times N$ matrix $\mathbf{B}$ are $b_{i j}=b>0$ and $b_{i i}=\underline{b}$, $i, j=1, \ldots, n, i \neq j$. Without loss of generality, we normalize $b=1$. Notice that $U$ is concave, homogeneous of degree 1 , and strictly monotonic if also $\underline{b}>0$. If only $n<N$ varieties are actually available it reduces to:

$$
U^{n}(\mathbf{x})=\sum_{i=1}^{n} \sum_{j=1}^{n} b_{i j} \sqrt{x_{i} x_{j}}
$$

whose functional form once again does not depend on $n$. Notice that utility becomes $U=n x(n-1+\underline{b})$ in the case of a symmetric consumption, which requires $n-1>-\underline{b}$. Computation shows that $\mu^{n}(\mathbf{x})=-U^{n}(\mathbf{x})$ by homogeneity, and:

$$
h^{n}\left(x_{i}, \mathbf{x}\right)=\underline{b}-1+\frac{1}{\sqrt{x_{i}}} \sum_{j=1}^{n} \sqrt{x_{j}}, \text { and } h_{1}^{n}\left(x_{i}, \mathbf{x}\right)=-\frac{x_{i}^{-3 / 2}}{2} \sum_{j=1}^{n} \sqrt{x_{j}} .
$$

Restricting the attention to the case in which $h>0$ (which always holds if $\underline{b}>0$ ), we obtain:

$$
\epsilon^{n}\left(x_{i}, \mathbf{x}\right)=\frac{\sum_{j=1}^{n} \sqrt{x_{j}}}{2 \sum_{j=1}^{n} b_{i j} \sqrt{x_{j}}}
$$

Notice that in a symmetric equilibrium:

$$
\epsilon^{n}=\frac{n}{2(n-1+\underline{b})},
$$

which satisfies $\partial \epsilon^{n} / \partial n<0$ iff $1>\underline{b}{ }^{11}$ An increase of $n$ produces an anticompetitive effect if $1<\underline{b}$, and in any case we have $\lim _{n \rightarrow \infty} \epsilon^{n}=1 / 2$, i.e. the markup would never become negligible even if $n$ was very large.

In the specification with $\underline{b}=1$ the markup becomes independent from the number of goods under monopolistic competition, because the Morishima elasticity of substitution is a constant. While CES preferences are the only separable preferences that induce constant markups, our result shows that there are other non-separable preferences that induce the same result. ${ }^{12}$ As usual, we can also derive the full set of imperfect competition equilibria:

$$
p=2 c, \quad p^{b}=\frac{(2 n-1) c}{n-1} \quad \text { and } \quad p^{c}=\frac{2 n c}{n-1} .
$$

\footnotetext{
${ }^{11}$ Here $\epsilon^{n}<1$ requires that $n>2(1-b)$.

${ }^{12} \mathrm{~A}$ generalization of $(25)$ as $U^{n}=\sum_{i_{1}=1}^{n} \cdots \sum_{i_{m}=1}^{n}\left(x_{i_{1}} \cdots x_{i_{m}}\right)^{1 / m}$ with $m \leq n$ would lead, under monopolistic competition, to the constant markup $(p-c) / c=1-1 / \mathrm{m}$.
} 
The general case provides interesting results, with markups that can increase in the number of firms not only under monopolistic competition, but also with strategic interactions. Under monopolistic competition the general markup is:

$$
\frac{p-c}{p}=\frac{n}{2(n-1+\underline{b})}
$$

which is increasing in the number of firms if and only if $\underline{b}>1$. Strategic interactions add a competition effect, that however does not necessarily compensate the anti-competitive effect inheritated through $\epsilon^{n}$. Indeed, under Cournot and Bertrand competition we can compute:

$$
\frac{p^{c}-c}{p^{c}}=\frac{n^{2}+n-2+2 \underline{b}}{2 n^{2}-2 n+n 2 \underline{b}} \quad \text { and } \quad \frac{p^{b}-c}{p^{b}}=\frac{n^{2}}{2 n^{2}-3 n+2+2(n-1) \underline{b}},
$$

which might be both increasing in the number of firms if this is large enough. ${ }^{13}$ As far as we know, this is the first computable example of Bertrand and Cournot equilibria with prices increasing with the number of firms. Notice, however, that profits remain decreasing in the number of firms, which will allow us to analyze endogenous market structures later on.

\subsection{Quadratic direct utility}

We now move to the general field of non-homothetic preferences that are also non-separable. Let us consider the following utility function:

$$
U^{n}(\mathbf{x})=\alpha \sum_{j=1}^{n} x_{j}-\frac{\gamma}{2} \sum_{j=1}^{n} x_{j}^{2}-\frac{\eta}{2}\left(\sum_{j=1}^{n} x_{j}\right)^{2}
$$

where $\alpha, \gamma, \eta>0$ : this corresponds to the non-linear part of the utility function used in Ottaviano and Thisse (1999) and later popularized by Melitz and Ottaviano (2008). ${ }^{14}$ The functional form of $U^{n}(\mathbf{x})$ does not change with changes in the number $n$ of available varieties, and its derivatives are given by $h^{n}\left(x_{i}, \mathbf{x}\right)=$ $\alpha-\gamma x_{i}-\eta \sum_{j=1}^{n} x_{j}$. Accordingly, the demand function perceived under monopolistic competition is the usual linear demand with $h_{1}^{n}\left(x_{i}, \mathbf{x}\right)=-\gamma$. It is easy to show that $U^{n}(\mathbf{x})$ is concave, and we restrict our attention to the case in which $h^{n}\left(x_{i}, \mathbf{x}\right)>0$ for any $i=1, \ldots, n$, and so it is also strictly monotonic.

\footnotetext{
${ }^{13}$ Under Cournot competition the markup is increasing if and only if:

$$
(2 n+1)\left[n^{2}-n+n \underline{b}\right]>\left[n^{2}+n-2+2 \underline{b}\right][n-1+\underline{b}],
$$

which simplifies to $(\underline{b}-2) n^{2}>4 n(\underline{b}-1)+2 \underline{b}^{2}-4 \underline{b}+2$. Under Bertrand competition if:

$$
2 n\left[2 n^{2}-3 n+2+2(n-1) \underline{b}\right]>n^{2}[4 n-3+2 \underline{b}],
$$

that is $n(2 \underline{b}-3)>4(\underline{b}-1)$. For instance, for $\underline{b}=3$ the price is increasing in the number of firms if $n>4+\sqrt{24}$ under Cournot competition and $n>8 / 3$ under Bertrand competition.

${ }^{14}$ See also Vives (1999, Ch. 6).
} 
The inverse demand function for variety $i$ is given by:

$$
s_{i}=\frac{\alpha-\gamma x_{i}-\eta \sum_{j=1}^{n} x_{j}}{\alpha \sum_{j=1}^{n} x_{j}-\gamma \sum_{j=1}^{n} x_{j}^{2}-\eta\left(\sum_{j=1}^{n} x_{j}\right)^{2}},
$$

with perceived demand elasticity:

$$
\epsilon^{n}\left(x_{i}, \mathbf{x}\right)=\frac{\gamma x_{i}}{h^{n}\left(x_{i}, \mathbf{x}\right)} .
$$

In the symmetric equilibrium we obtain:

$$
\epsilon^{n}(x)=\frac{\gamma x}{\alpha-\gamma x-n \eta x},
$$

with $\partial \epsilon^{n} / \partial n, \partial \epsilon^{n} / \partial x>0, \lim _{x \rightarrow 0} \epsilon^{n}(x)=0$ and $\lim _{x \rightarrow \alpha /(2 \gamma+n \eta)} \epsilon^{n}(x)=1 .{ }^{15}$ Therefore we have:

$$
p=\frac{c}{1-\epsilon^{n}(x)}=m(x, n) c,
$$

where $m=h^{n}(x) /(\alpha-2 \gamma x-n \eta x), m(0, n)=1$ and $\partial \ln m / \partial \ln x>\partial \ln m / \partial \ln n>$ 0 . Since a monopolistic competition equilibrium is then characterized by:

$$
\frac{E}{n}=m(x, n) x c,
$$

we can be sure that such an equilibrium does exist and it is unique. Notice that (33) implies $\partial x / \partial E>0$ and $\partial x / \partial n<0$, and accordingly $\partial p / \partial E>0$ and $\partial p / \partial n<0$ : a seemingly competitive effect emerges even in the absence of strategic interactions (and in spite of the fact that $\partial m / \partial n>0$ ) ${ }^{16}$ Similarly, we get:

$$
p^{c}=\frac{n m\left(x^{c}, n\right) c}{(n-1)} \quad \text { and } \quad p^{b}=\frac{\left[n m\left(x^{b}, n\right)-1\right] c}{(n-1)} .
$$

under Cournot and Bertrand competition.

\subsection{Generalized quadratic preferences}

We finally introduce a last class of preferences that has not been fully explored (as far as we know) in the literature on imperfect competition, characterized by a quadratic indirect utility:

$$
V(\mathbf{s})=\mathbf{s}^{\prime} \boldsymbol{\beta} \mathbf{s}-\boldsymbol{\alpha}^{\prime} \mathbf{s}
$$

\footnotetext{
${ }^{15}$ Accordingly, $\epsilon^{n}<1$ requires $x<\alpha /(2 \gamma+n \eta)$. Incidentally, notice that $\gamma \rightarrow 0$ leads to marginal cost pricing (as goods tend to become perfect substitutes and monopolistic competition tends to perfect competition).

${ }^{16} \mathrm{It}$ is easily proved that:

$$
\frac{d m}{d n}=\frac{\partial m}{\partial n}+\frac{\partial m}{\partial x} \frac{\partial x}{\partial n}<o
$$

iff $\partial \ln m / \partial \ln x>\partial \ln m / \partial \ln n$.
} 
where $\boldsymbol{\beta}$ is a $N \times N$ matrix and $\boldsymbol{\alpha}$ a $N$ vector, and $N$ is as usual the given total number of potential varieties. To achieve symmetry we assume that $\alpha_{j}=\alpha>0$, $\beta_{i j}=-b<0$ and $\beta_{i i}=\underline{\beta}, i, j=1, \ldots, n, i \neq j$. Normalizing $\underline{\beta}=b(N-1)>0$, Appendix D shows that $\bar{V}$ is convex ( $\boldsymbol{\beta}$ is a positive semidefinite matrix), and monotonic decreasing in the case $\alpha \boldsymbol{\iota} \geq 2 \boldsymbol{\beta}$ s, to which we restrict our attention. Moreover, preferences over the $n$ available varieties can be expressed as:

$$
\begin{aligned}
V^{n}(\mathbf{s}) & =\frac{N-n}{n \beta} \frac{\alpha^{2}}{4}+\sum_{i=1}^{n} \sum_{j=1}^{n} \beta_{i j} s_{i} s_{j}-\frac{\alpha N}{n} \sum_{j=1}^{n} s_{j}+\frac{N-n}{n} \beta\left(\sum_{j=1}^{n} s_{j}\right)^{2} \\
& =a_{0}+\sum_{j=1}^{n}\left[\underline{b} s_{j}^{2}-b s_{j} \sum_{k \neq j}^{n} s_{k}-a s_{j}\right]-d\left(\sum_{j=1}^{n} s_{j}\right)^{2}
\end{aligned}
$$

where $a_{0}=-\frac{N-n}{n b} \frac{\alpha^{2}}{4}, a=\frac{\alpha N}{n}$ and $d=\frac{N-n}{n} b$. The individual demand function has thus the linear form:

$$
x_{i}=\frac{a-2 b N\left(s_{i}-\underline{s}\right)}{-\mu^{n}(\mathbf{s})} \text { with } a=\frac{\alpha N}{n},
$$

where $\underline{s}=\sum_{j=1}^{n} s_{j} / n$ is the average price. It is remarkable that, when we properly take into account the prices of the unavailable varieties, the direct demand is perceived as linear in the difference between the own price and the average price of the available varieties, so reproducing the typical form adopted in textbook examples of monopolistic competition (see e.g. Vives, 1999, Ch. 6).

Since $g^{n}\left(s_{i}, \mathbf{s}\right)=2 b N\left(s_{i}-\underline{s}\right)-a$, the demand elasticity perceived in monopolistic competition is given by:

$$
\varepsilon^{n}(\mathbf{s})=\frac{2 b N s_{i}}{a-2 b N\left(s_{i}-\underline{s}\right)},
$$

which becomes

$$
\varepsilon^{n}(s)=\frac{2 b n s}{\alpha}
$$

in a symmetric equilibrium, after using $a=\alpha N / n .{ }^{17}$ Notice that $\varepsilon^{n}$ increases with $n s,{ }^{18}$ and we can obtain the three equilibrium markups. Solving for the symmetric equilibrium prices we have:

$$
p=c+\frac{\alpha E}{2 b n}, \quad p^{b}=\frac{\Phi+\sqrt[2]{\Phi^{2}+\frac{8 n c b \alpha E}{n-1}}}{4 n b} \quad \text { and } \quad p^{c}=\frac{n c}{(n-1)}+\frac{\alpha E}{2 b n} .
$$

where $\Phi \equiv \alpha E+2 n c b$. Prices increases in income and decrease in the number of goods in all cases.

\footnotetext{
${ }^{17}$ Note that a comparison with (31) shows that preferences (36) and (30) are actually different.

${ }^{18}$ Under our assumptions, $\varepsilon^{n}>1$ then requires $n s>\alpha / 2 b$.
} 


\section{Endogenous Market Structures}

We now consider the impact of free entry of firms. The symmetric equilibrium price is implicitly defined as a function of the number of firms by:

$$
\frac{p-c}{p}=\frac{1}{\theta(p / E, n)}
$$

where the function $\theta(s, n)>1$ depends on the form of competition. In particular, we have,

$$
\theta(s, n)=\varepsilon^{n}(s)=\frac{1}{\epsilon^{n}(1 / s n)}
$$

under monopolistic competition,

$$
\theta(s, n)=\frac{n \varepsilon^{n}(s)}{\varepsilon^{n}(s)+n-1}=\frac{n}{1+\epsilon^{n}(1 / s n)(n-1)}
$$

under Cournot competition, and

$$
\theta(s, n)=\frac{1+(n-1) \varepsilon^{n}(s)}{n}=\frac{\epsilon^{n}(1 / s n)+(n-1)}{n \epsilon^{n}(1 / s n)}
$$

under Bertrand competition. The total differentiation of the markup rule provides:

$$
\frac{d \ln s}{d \ln n}=\frac{-\psi_{n}}{\theta-1+\psi_{s}}
$$

where we defined $\psi_{s} \equiv \partial \ln \theta / \partial \ln s$ and $\psi_{n} \equiv \partial \ln \theta / \partial \ln n$ as the elasticities of $\theta(s, n)$ with respect to price and number of firms.

In what follows we make two "regularity assumptions". The first one is that $A \equiv \theta-1+\psi_{s}>0$, which implies that the impact of the number of firms on prices has a sign opposite to that of its impact on the relevant price elasticity. ${ }^{19}$ Since the equilibrium profit of each firm, $\pi=E L / n \theta(s(n), n)-F$, decreases with respect to $n$ if and only if:

$$
\begin{aligned}
\frac{d \ln n \theta(s(n), n)}{d n} & =1+\psi_{n}+\psi_{s} \frac{d \ln s}{d \ln n}>0 \\
& \Longleftrightarrow \quad D=[\theta-1]\left(1+\psi_{n}\right)+\psi_{s}>0
\end{aligned}
$$

our second "regularity assumption" is that $D>0 .{ }^{20}$ The endogenous-entry equilibrium conditions can then be expressed as:

$$
\frac{p-c}{p}=\frac{1}{\theta(p / E, n)} \quad \text { and } \quad n=\frac{E L}{F \theta(p / E, n)} .
$$

\footnotetext{
${ }^{19}$ Notice that $A>0$ will be certainly satisfied if $\psi_{s}>0$.

${ }^{20}$ Our assumptions are not independent: given $A>0, D$ will be positive unless $\psi_{n}$ is negative and "large" (in absolute value).
} 
Notice that these conditions (41) can be re-arranged to show a positive and convex relation between the price and the number of firms:

$$
p=\frac{c E L}{E L-n F} .
$$

Let us assume that the free-entry equilibrium is unique and well-defined. The first result we can obtain is a complete ranking of the number of firms and equilibrium markups emerging in the three forms of competition, as an immediate consequence of Corollary 4 and the positive relation between price and number of firms just stated:

Proposition 5 If $\varepsilon^{n}>1\left(\epsilon^{n}<1\right)$ everywhere, under endogenous market structures the equilibrium number of firms and markup under Cournot competition are higher than those under Bertrand competition, which are higher than those under generalized monopolistic competition.

The total differentiation of the equilibrium system (41) provides the following elasticities of the equilibrium variables with respect to income $E$, market size $L$ and marginal cost $c$ :

$$
\begin{aligned}
& \frac{d \ln p}{d \ln E}=\frac{\psi_{s}-\psi_{n}}{D} \quad \text { and } \quad \frac{d \ln n}{d \ln E}=\frac{\theta-1+\theta \psi_{s}}{D}, \\
& \frac{d \ln p}{d \ln L}=\frac{-\psi_{n}}{D} \quad \text { and } \quad \frac{d \ln n}{d \ln L}=\frac{\theta-1+\psi_{s}}{D} \text {, } \\
& \frac{d \ln p}{d \ln c}=1-\frac{\psi_{s}}{D} \quad \text { and } \quad \frac{d \ln n}{d \ln c}=\frac{-(\theta-1) \psi_{s}}{D} .
\end{aligned}
$$

Let us define neutrality on the market structure of income $E$, of the market size $L$ and of the marginal cost $c$ as the property for which a change in one of these parameters does not affect the equilibrium markup. The comparative statics in (43)-(45) implies that the market size is neutral on the endogenous market structure if and only if $\psi_{n}=0$, income is neutral if and only if $\psi_{s}=\psi_{n}$ and the marginal cost is neutral if and only if $\psi_{s}=0$. The implications for our examples are immediate under monopolistic competition: 1) the neutrality of the market size holds not only with CES preferences (Krugman, 1980) but under any case of indirect additivity (Bertoletti and Etro, 2013): in these cases a market that is twice as large attracts a double number of firms selling the same quantity at the same price and generating pure gains from variety; 2) the neutrality of income holds under direct additivity (see Zhelobodko et al., 2012 and Bertoletti and Etro, 2014) and even in the case of the Generalized quadratic preferences: in these cases a double income attracts a double number of firms; and 3) the neutrality of the marginal cost holds under any homothetic preferences, so that changes in productivity are translated proportionally on prices and do not affect the number of firms. The three neutralities hold at the same time under CES preferences and also in our example of Generalized linear preferences with equal coefficients $\left(b_{i j}=1\right)$. 
Similarly, let us say that an increase of income, market size or productivity exerts a competition effect if it reduces the markups. Notice that such an effect is associated in the first two cases with an increase less than proportional of the number of firms, and in the third case with a reduction of it (a case of so-called "business destruction"). The next result summarizes the general comparative statics of the free-entry equilibrium:

Proposition 6 Under endogenous market structures: 1) an increase in the market size exerts a competition effect if and only if $\psi_{n}>0$; 2) an increase in income exerts a competition effect if and only if $\psi_{s}<\psi_{n}$; 3) an increase in productivity exerts a competition effect if and only if $\psi_{s}>0$.

The implications for the case of monopolistic competition are again particularly tranchant. To exemplify our result, let us consider the case of a quadratic direct utility, in which none of the mentioned three neutralities holds. From (31) we can obtain $\theta=(\alpha s-\eta) n / \gamma-1$, which implies: ${ }^{21}$

$$
\psi_{s}=\frac{\alpha s n}{\alpha s n-\eta n-\gamma}>\psi_{n}=\frac{\alpha s n-\eta n}{\alpha s n-\eta n-\gamma}>0 .
$$

Accordingly, under free entry an increase in market size or in productivity reduces markups, but an increase in income increases prices (and expands the number of firms more than proportionally).

Similar applications of the first result of Proposition 6 can be done to our other examples. In particular, market size exerts a competition effect under direct additivity when the elasticity of substitution decreases with consumption, while an anti-competitive effect arises if the elasticity of substitution is increasing in consumption (see Bertoletti et al., 2008 and Zhelobodko et al., 2012). A competition effect shows up also in the Translog case, under the Generalized Leontief preferences and in the case of Generalized quadratic preferences. Finally, Generalized linear preferences can generate either a competitive or an anti-competitive effect of the market size.

The second result of Proposition 6 shows that the competitive effect due to an increase in income arises only if $\psi_{n}-\psi_{s}>0$ : clearly, this is a condition which can be satisfied under several cases. Bertoletti and Etro (2013) obtain a competition effect of income under indirect additivity with demand elasticity decreasing with respect to prices $\left(\psi_{n}=0, \psi_{s}<0\right)$, but the same happens under homotheticity $\left(\psi_{s}=0\right)$ as long as $\psi_{n}>0$, which holds in the Translog and Generalized Leontief preferences cases. Nevertheless, markups increasing in income are much more in line with the trade evidence on pricing to market (for instance see Simonovska, 2013) or some macroeconomic evidence on procyclical markups (Nekarda and Ramey, 2013): a positive relation between income and markups emerges under indirect additivity with demand elasticity increasing in the price-income ratio $\left(\psi_{n}=0, \psi_{s}>0\right)$.

\footnotetext{
${ }^{21}$ Notice that $\theta>1$ implies $\alpha s>\eta$.
} 
The third result of Proposition 6 shows that the price sensitivity of demand elasticity is what matters to determine the translation of marginal costs (including taxes or any other cost wedge) on prices: in the (arguably) more realistic case in which demand is perceived as more rigid when income increases $\left(\psi_{s}>0\right)$, changes in productivity are translated less than proportionally on prices, and additional costs are undershifted. This is for instance what happens in both of our quadratic examples.

Moving to the case of oligopolistic competition increases the value of $\psi_{n}$, thus raising the chances of getting competition effects due to market size or income. Consider for instance an increase in the market size: even when a larger market size raises markups under monopolistic competition, strategic interactions may give rise to a competition effect on the markups, at least when the number of firms is small. A case in which we can state unambiguous results is the one of homothetic preferences, since markups depend only on the number of goods:

Corollary 7 Under homotheticity, endogenous market structures generate neutrality of productivity, while income and market size exert a competition effect if and only if $\psi_{n}>0$.

The case of homothetic preferences is particularly relevant in dynamic (macroeconomic) applications where homotheticity of the consumption index is crucial for two-stage budgeting. Bilbiie et al. (2012) have indeed adopted homothetic preferences in a monopolistic competition model with endogenous entry to study the impact of aggregate shocks. These shocks can be magnified through competition effects in case of demand elasticity increasing with entry, as with the Translog preferences adopted by Bilbiie et al. (2012) or with the introduction of strategic interactions à la Bertrand or Cournot as in Etro and Colciago (2010).

To conclude this section with a few examples, let us return to the Feenstra (2003) example of Translog preferences. Under monopolistic competition we can solve for the free entry number of goods as:

$$
n=\sqrt{\frac{E L}{\gamma F}+\left(\frac{1}{2 \gamma}\right)^{2}}-\frac{1}{2 \gamma}
$$

This implies that both income and market size affect the market structure: an increase in $E L / F$ attracts more firms, which reduces markups. Moreover, an increase of $\gamma$ reduces product differentiation, thereby decreasing markups and the equilibrium number of firms. The same happens under Bertrand and Cournot competition, which yield:

$$
n^{c}=\sqrt{\frac{E L(1+\gamma)}{\gamma F}+\frac{1}{4 \gamma^{2}}}-\frac{1}{2 \gamma}>n^{b}=\sqrt{\frac{E L}{\gamma F}+\left(\frac{1-\gamma}{2 \gamma}\right)^{2}}-\frac{1-\gamma}{2 \gamma}>n .
$$

Similar competition effects are associated to the case of the Generalized Leontief preferences for which we obtain:

$$
n=\sqrt{\frac{2 E L}{F}}
$$


The case of Generalized linear preferences delivers the following equilibrium under monopolistic competition:

$$
n=\frac{E L}{2 F}+1-\underline{b},
$$

which is exactly linear in $E L / F$ only if $\underline{b}=1$ and thus $\psi_{n}=0$.

Finally, let us consider our examples of non-homothetic and non-separable preferences. In case of quadratic direct utility the closed form solution is complex but we have already discussed its comparative statics. Under the Generalized quadratic preferences the equilibrium number of firm with monopolistic competition is given by:

$$
n=\frac{\alpha E\left(\sqrt{1+\frac{8 b c L}{\alpha F}}-1\right)}{4 b c},
$$

which shows a linear relation with income and a concave one in population.

\section{Optimal Market Structures}

The optimal organization of production can be reached by a social planner controlling number of firms, production and prices. ${ }^{22}$ Such an optimal market structure can be identified maximizing utility under the resource constraint $E L=n c x L+n F$. This is equivalent to the use of the budget and the zero profit constraints, which can be combined to obtain (42). Let us define $U(x ; n)=$ $U^{n}(x \boldsymbol{\iota})$ and equivalently $V(s ; n)=V^{n}(s \boldsymbol{\iota})$. We can now state the social planner problem as the maximization of:

$$
W(n)=U\left[\frac{E}{c n}-\frac{F}{c L} ; n\right] \quad \text { or } W(n)=V\left[\frac{c L}{E L-F n} ; n\right] .
$$

Let us define $\phi(x ; n) \equiv U_{x}(x ; n) x / U(x ; n)>0$ as the elasticity of the direct utility with respect to the consumption level and $\varphi(x ; n) \equiv U_{n}(x ; n) n / U(x ; n)>$ 0 as the elasticity of the direct utility with respect to the number of varieties. Notice that $\phi(x ; n)$ is just the so-called "scale elasticity" of $U^{n}$ at $x \boldsymbol{\iota}$. Differentiating the equality $U(E / p n ; n)=V(p / E ; n)$ we obtain that $\phi=$ $-V_{s}(s ; n) s / V(s ; n)$ is also the elasticity of the "corresponding" indirect utility with respect to the price of each good, and $\widetilde{\varphi}=\varphi-\phi=V_{n}(s ; n) n / V(s ; n)$ is the elasticity of the "corresponding" indirect utility with respect to the number of available varieties. We can now express the conditions for the optimal provision of goods in the following result:

Proposition 8 The optimal market structure requires

$$
p^{*}=c \frac{\varphi}{\phi} \quad \text { and } \quad n^{*}=\frac{E L(1-\phi / \varphi)}{F},
$$

\footnotetext{
${ }^{22}$ On the optimal market structures see the original contribution by Dixit and Stiglitz (1977) and the extensions in Kuhn and Vives (1999) and more recently Nocco et al. (2014) and Mrázová and Neary (2014).
} 
where $\phi$ is the elasticity of direct utility with respect to the consumption of each good and $\varphi$ the elasticity of direct utility with respect to the number of varieties.

The rule (51) implies that prices and number of goods provided should be increasing in $\varphi$, due to larger benefits from variety, and decreasing in $\phi$, due to larger benefits of higher consumption of each good. The optimal market structures under additivity have been characterized by Dixit and Stiglitz (1977) for direct additivity $(\varphi=1)$ and Bertoletti and Etro (2013) for indirect additivity $(\widetilde{\varphi}=1)$. Under monopolistic competition and additivity free entry is optimal only under CES preferences $(\varphi / \phi=\theta /(\theta-1))$.

Benassy (1996) has suggested that homotheticity can generate both excess and insufficient free entry. Indeed, notice that under homotheticity the scale of consumption does not matter for the optimal choice, and normalizing the direct utility to be homogenous of degree 1 we get $\phi=1$ and $U(x ; n)=x U^{n}(\iota)=$ $n t(n) x$, with $\varphi(n)=1+\chi(n)$, where $\chi(n)=d \ln t(n) / d \ln n$ it is what Benassy (1996) called the "taste for variety" embedded into preferences. It follows from (51), (42) and (41) that a sufficient condition for excess (insufficient) entry under homothetic preferences is $\chi(n)<(>) 1 /(\theta(n)-1) .{ }^{23}$ Analogously, we can write $V(s ; n)=V^{n}(\iota) / s=v(n) / s$, with $\widetilde{\varphi}(n)=d \ln v(n) / d \ln n$, and the sufficient condition for excess (insufficient) entry becomes $\widetilde{\varphi}(n)<(>) 1 /(\theta(n)-1)$.

In the case of Translog preferences we actually have:

$$
V(s ; n)=\frac{1}{s} \exp \left[\frac{1}{2 \gamma N}-\alpha_{0}-\frac{1}{2 \gamma n}\right],
$$

with

$$
\widetilde{\varphi}(n)=\frac{1}{2 \gamma n}<\frac{1}{\gamma n}=\frac{1}{\varepsilon^{n}-1} .
$$

This shows that the free-entry equilibrium number of firms under monopolistic competition (46) is characterized by excess entry, as can be verified comparing it with the optimal one:

$$
n^{*}=\sqrt{\frac{E L}{2 \gamma F}+\left(\frac{1}{4 \gamma}\right)^{2}}-\frac{1}{4 \gamma} .
$$

A fortiori the number of firms is further away from the optimal one moving to Bertrand competition and even more when moving to Cournot competition.

In case of Generalized Leontief preferences we obtain the following expression (see Appendix C):

$$
V(s ; n)=\frac{n-1}{\operatorname{sn}(N-1)},
$$

with

$$
\widetilde{\varphi}(n)=\frac{1}{n-1}<\frac{2}{n-2}=\frac{1}{\varepsilon^{n}-1},
$$

\footnotetext{
${ }^{23}$ In addition, it can be shown that $\epsilon^{n}$ takes a particular simple expression in the case of homothetic preferences, given by $n U_{j i}^{n}(\iota) / U_{i}^{n}(\iota)$.
} 
showing that again excess entry occurs with imperfect competition. The optimal number can be computed as:

$$
n^{*}=\frac{n}{\sqrt{2}},
$$

where $n$ is given by (47).

Let us move to the Generalized linear preferences, which imply:

$$
U(x ; n)=n x(n-1+\underline{b}) .
$$

Since

$$
\chi(n)=\frac{n}{n-1+\underline{b}}<\frac{n}{n-2+2 \underline{b}}=\frac{\epsilon^{n}}{1-\epsilon^{n}},
$$

excess entry occurs under monopolistic competition when the markup is decreasing in the number of firms $(\underline{b}<1)$ and insufficient entry arises otherwise $(\underline{b}>1)$. Indeed, the optimal number of firms can be computed as:

$$
n^{*}=n-\frac{1-\underline{b}}{2},
$$

where $n$ is given by (48).

In the case of quadratic direct utility we have:

$$
U(x ; n)=n x\left[\alpha-\frac{x}{2}(\gamma+\eta n)\right]
$$

and entry is generally inefficient. ${ }^{24}$ Finally, under Generalized quadratic preferences we can derive (see Appendix D):

$$
V(s ; n)=-\frac{N-n}{n b} \frac{\alpha^{2}}{4}-\alpha N s .
$$

whose maximization implies that excess entry arises always under free entry. ${ }^{25}$

\section{Conclusion}

We have characterized imperfect competition with demand functions derived from symmetric preferences, discussing some general results and new examples. The symmetric equilibrium markups depend on the elasticity of substitution/complementarity between goods, which corresponds to the demand elasticity perceived by firms under monopolistic competition, and can be derived

\footnotetext{
${ }^{24}$ See Ottaviano and Thisse (1999) and Nocco et al. (2014) on a related analysis with quasilinear preferences.

${ }^{25}$ Indeed, the free entry equilibrium implies $(p-c)^{2}=\frac{\alpha F}{2 b L} p$, while using the zero profit constraint $n=(p-c) E L / p F$, the optimum solves:

$$
\max _{p} \frac{-\alpha p F}{4(p-c) b L}-p
$$

with FOC $\left(p^{*}-c\right)^{2}=\frac{\alpha F}{2 b L} \frac{c}{2}$. Since $p>c / 2$ it must be that $p>p^{*}$.
} 
directly from the direct or indirect utility function. Our examples show that a rich set of competitive/anticompetitive effects can arise when the number of varieties changes, or there is endogenous entry. Notably, in the latter case, three well-known classes of preferences (namely, direct and indirect additive preferences, and homothetic preferences) partially neutralize the impact of individual income, market size and marginal cost on the equilibrium markups. In the general case, on the contrary, all these do affect the equilibrium values. Finally, the free-entry equilibrium can exhibit either excess or insufficient entry with respect to the optimal market allocation.

Future research could apply this setting to reconsider a number of industrial organization issues, such as the welfare properties of the different equilibria, the impact of mergers or the role of market leaders and strategic commitments with endogenous market structures (see for instance Etro, 2011). It would also be interesting to introduce endogenous sunk costs to generalize the result by Sutton (1991) on the non-monotone relation between market size and number of firms (see also Vives, 2008). Finally, in terms of applications, the framework we propose could be used to investigate the robustness of the results generated in trade and macroeconomic models by the standard monopolistic competition cum CES preferences. ${ }^{26}$

\section{References}

Anderson, S., N. Erkal and D. Piccinin, 2012, Aggregative Games and Entry, mimeo, University of Virginia

Benassy, J.-P., 1996, Taste for Variety and Optimum Production patterns in Monopolistic Competition, Economics Letters, 52, 1, 41-47

Bertoletti, P., 2005, Elasticities of Substitution and Complementarity: A Synthesis, Journal of Productivity Analysis, 24, 2, 181-94

Bertoletti, P., E. Fumagalli and C. Poletti, 2008, Price-Increasing Monopolistic Competition? The Case of IES Preferences," IEFE Working Paper No. 15, Bocconi University.

Bertoletti, P. and F. Etro, 2013, Monopolistic Competition when Income Matters, DEM WP55, University of Pavia, Department of Economics and Management

Bertoletti, P. and F. Etro, 2014, Pricing to Market in the Krugman Model, Economics Bulletin, 34, 1, 459-68

Bertoletti, P. and P. Epifani, 2014, Monopolistic Competition: CES Redux?, Journal of International Economics, 93, 2, 227-38

Bilbiie, F., F. Ghironi and M. Melitz, 2012, Endogenous Entry, Product Variety, and Business Cycles, Journal of Political Economy, 120, 2, 304-45

\footnotetext{
${ }^{26}$ For recent steps in this direction, Bilbiie et al. (2012) and Etro and Colciago (2010) have introduced respectively Translog preferences and strategic interactions in macroeconomic models with endogenous entry, and Etro (2015) has introduced strategic interactions in a $2 \times 2 \times 2$ trade model.
} 
Blackorby, C. and R. Russell, 1981, The Morishima Elasticity of Substitution: Symmetry, Constancy, Separability, and its Relationship to the Hicks and Allen Elasticities, Review of Economic Studies, 48, 147-58

Blackorby, C. and R. Russell, 1989, Will the Real Elasticicity of Substitution Plase Stand Up? (A Comparison of the Allen/Uzawa and Morishima Elasticities), The American Economic Review, 79, 882-8

Campbell, J. and H. Hopenhayn, 2005, Market Size Matters, Journal of Industrial Economics, 53, 1, 1-25

Diewert, W., 1971, An Application of the Shephard Duality Theorem: A Generalized Leontief Production Function, Journal of Political Economy, 79, 3, 481-507

Dixit, A. and J. Stiglitz, 1977, Monopolistic Competition and Optimum Product Diversity, The American Economic Review, 67, 297-308

Etro, F., 2011, Endogenous Market Structures and Contract Theory: Delegation, Principal-Agent Contracts, Screening, Franchising and Tying, European Economic Review, 55, 4, 63-79

Etro, F., 2015, Endogenous Market Structures and International Trade, Scandinavian Journal of Economics, in press

Etro, F. and A. Colciago, 2010, Endogenous Market Structures and the Business Cycle, The Economic Journal, 120, 1201-34

Feenstra, R., 2003, A Homothetic Utility Function for Monopolistic Competition Models without Constant Price Elasticity, Economics Letters, 78, 1, 79-86

Hanoch, G., 1974, Production and Demand Models with Direct or Indirect Implicit Additivity, Econometrica, 43, 3, 395-419

Kuhn, K.-U. and X. Vives, 1999, Excess Entry, Vertical Integration, and Welfare, RAND Journal of Economics, 30, 4, 575-603

Krugman, P., 1980, Scale Economies, Product Differentiation, and the Pattern of Trade, The American Economic Review, 70, 950-9

Melitz, M., 2003, The Impact of Trade on Intra-Industry Reallocations and Aggregate Industry Productivity, Econometrica, 71, 6, 1695-725

Melitz, M. and G. Ottaviano, 2008, Market Size, Trade, and Productivity, Review of Economic Studies 75, 1, 295-316

Mrázová, M. and P. Neary, 2014, Together at Last: Trade Costs, Demand Structure, and Welfare, American Economic Review, 104, 5, 298-303

Mundlak, Y., 1968, Elasticity of Substitution and the Theory of Derived Demand, Review of Economic Studies, 35, 225-36

Nekarda, C. and V. Ramey, 2013, The Cyclical Behavior of the Price-Cost Markup, NBER WP 19099

Nocco, A., G. Ottaviano and M. Salto, 2014, Monopolistic Competition and Optimum Product Selection, American Economic Review, 104, 5, 304-09

Ottaviano, G. and J.-F. Thisse, 1999, Monopolistic Competition, Multiproduct Firms and Optimum Product Diversity, CEPR DP 2151

Pollak, R., 1972, Generalized Separability, Econometrica, 40, 3, 431-53

Sherman, J. and W. Morrison, 1950, Adjustment of an Inverse Matrix Corresponding to a Change in One Element of a Given Matrix, Annals of Mathematical Statistics, 21, 1, 124-7 
Simonovska, I., 2013, Income Differences and Prices of Tradables, mimeo, University of California, Davis

Spence, A. M., 1976, Product Selection, Fixed Costs, and Monopolistic Competition, Review of Economic Studies, 43, 217-35

Sutton, John, 1991, Sunk Costs and Market Structure: Price Competition, Advertising, and the Evolution of Concentration, MIT Press, London

Varian, H., 1992, Microeconomic Analysis, Norton \& Company, New York

Vives, X., 1985, On the Efficiency of Bertrand and Cournot Equilibria with Product Differentation, Journal of Economic Theory, 36, 1, 166-75

Vives, X., 1999, Oligopoly Pricing. Old Ideas and New Tools, MIT Press, London

Vives, X., 2008, Innovation and Competitive Pressure, Journal of Industrial Economics, 56, 3, 419-69

Zhelobodko, E., S. Kokovin, M. Parenti and J.-F. Thisse, 2012, Monopolistic Competition in General Equilibrium: Beyond the CES, Econometrica, 80, 6, $2765-84$

\section{Appendix}

\section{A: Properties of Symmetric Functions}

Let us consider a twice-differentiable function $y=f(\mathbf{x})$ :

$$
f: \mathbb{R}_{+}^{n} \longrightarrow \mathbb{R}
$$

We say that $f$ is symmetric with respect to its arguments $\mathbf{x}$ if

$$
f(\mathbf{x})=f(\mathbf{P x}), \quad \forall \mathbf{x}, \forall \mathbf{P},
$$

where $\mathbf{P}$ is a permutation matrix. The symmetry of $f$ imposes some symmetry also on its derivatives. Define:

$$
f_{i}(\mathbf{x})=\frac{\partial f(\mathbf{x})}{\partial x_{i}} \text { and } f_{i j}(\mathbf{x})=\frac{\partial^{2} f(\mathbf{x})}{\partial x_{i} \partial x_{j}},
$$

$i, j=1, \ldots, n$. Differentiation of (55) with respect to $\mathbf{x}$ yields, in matrix notation:

$$
\operatorname{Df}(\mathbf{x})=\mathbf{P}^{\prime} \operatorname{Df}(\mathbf{P} \mathbf{x}) .
$$

Since $\mathbf{P}$ is orthogonal, i.e., $\mathbf{P}^{\prime}=\mathbf{P}^{-1}$, we obtain:

$$
\mathbf{P} D f(\mathbf{x})=D f(\mathbf{P} \mathbf{x}), \quad \forall \mathbf{x}, \forall \mathbf{P},
$$

which shows that the gradient of a symmetric scalar function is itself a symmetric vector function. Similarly, differentiating (56) with respect to $\mathbf{x}$ yields:

$$
\mathbf{P} D^{2} f(\mathbf{x})=D^{2} f(\mathbf{P x}) \mathbf{P}
$$

i.e.,

$$
\mathbf{P} D^{2} f(\mathbf{x}) \mathbf{P}^{\prime}=D^{2} f(\mathbf{P} \mathbf{x}), \quad \forall \mathbf{x}, \forall \mathbf{P} .
$$


(56) and (57) imply that:

$$
f_{i}(\mathbf{x})=\widetilde{g}\left(x_{i}, \mathbf{x}_{-i}\right),
$$

where $\widetilde{g}$, which does not depend on $i$, is symmetric with respect to $\mathbf{x}_{-i}$.

Indeed, it can be also proved that

$$
f_{i}(\mathbf{x})=g\left(x_{i}, \mathbf{x}\right),
$$

where $g$ is symmetric with respect to $\mathbf{x}$. The formal argument for this additional result was kindly provided by Giuseppe Savaré, of the Department of Mathematics of the University of Pavia. A skecth of the proof follows. First, for each symmetric function $f(\mathbf{x})$ it holds that

$$
f(\mathbf{x})=\widetilde{f}\left(\mathbf{S}^{n}(\mathbf{x})\right),
$$

where $\widetilde{f}$ is unique and well defined and

$$
\mathbf{S}_{m}^{n}(\mathbf{x})=\sum_{0<i_{1}<\ldots<i_{m} \leq m} \prod_{k=1}^{m} x_{i_{k}},
$$

with $m=1, \ldots, n$. Notice that each of the $n$ functions $\mathbf{S}_{m}^{n}$ is symmetric, and that any $\mathbf{x}$ is associated to a specific $\mathbf{S}^{n}$ up to a permutation. Second, since:

$$
\mathbf{S}_{k}^{n-1}\left(\mathbf{x}_{-i}\right)=\sum_{j=0}^{k}(-1)^{j} x_{i}^{j} \mathbf{S}_{k-j}^{n}(\mathbf{x}),
$$

where $\mathbf{S}_{0}^{n}=1$, it follows that:

$$
\widetilde{g}\left(x_{i}, \mathbf{x}_{-i}\right)=\widehat{g}\left(x_{i}, \mathbf{S}_{k}^{n-1}\left(\mathbf{x}_{-i}\right)\right)=g\left(x_{i}, \mathbf{x}\right),
$$

where $g$ is symmetric with respect to $\mathbf{x}$.

\section{B: Cournot and Bertrand symmetric equilibria}

Let us consider the "primal approach", based on the inverse demand system $\mathbf{s}(\mathbf{x})$. The Cournot equilibrium is found by direct calculation. To find the equilibrium under Bertrand competition we must obtain the elasticity of the direct demand from the inversion of the inverse demand system, which in matrix notation is implicitly defined by $\mathbf{p} \lambda(\mathbf{x})-\mathbf{f}(\mathbf{x})=0$, where $f_{i}=U_{i}^{n}, i=1, \ldots, n$ and $\lambda(\mathbf{x})=-\mu^{n}(\mathbf{x})$. By the Implicit Function Theorem we have:

$$
\mathbf{B} D_{\mathbf{p}} \mathbf{x}(\mathbf{p})=-\lambda(\mathbf{x}) \mathbf{I},
$$

where $\mathbf{I}$ is the relevant identity matrix and

$$
\mathbf{B}=\mathbf{p} D_{\mathbf{x}} \lambda(\mathbf{x})^{\prime}-D_{\mathbf{x}} \mathbf{f}(\mathbf{x})=\left[\begin{array}{ccc}
p_{1} \frac{\partial \lambda}{\partial x_{1}}-\frac{\partial f_{1}}{\partial x_{1}} & \ldots & p_{1} \frac{\partial \lambda}{\partial x_{n}}-\frac{\partial f_{1}}{\partial x_{n}} \\
\ldots & \ldots & \ldots \\
p_{n} \frac{\partial \lambda}{\partial x_{1}}-\frac{\partial f_{n}}{\partial x_{1}} & \ldots & p_{n} \frac{\partial \lambda}{\partial x_{n}}-\frac{\partial f_{n}}{\partial x_{n}}
\end{array}\right],
$$




$$
D_{\mathbf{p}} \mathbf{x}(\mathbf{p})=\left[\begin{array}{ccc}
\frac{\partial x_{1}}{\partial p_{1}} & \ldots & \frac{\partial x_{1}}{\partial p_{n}} \\
\ldots & \ldots & \ldots \\
\frac{\partial x_{n}}{\partial p_{1}} & \ldots & \frac{\partial x_{n}}{\partial p_{n}}
\end{array}\right] \text { and }-\lambda(\mathbf{x}) \mathbf{I}=\left[\begin{array}{ccc}
-\lambda & \ldots & 0 \\
\ldots & \ldots & \ldots \\
0 & \ldots & -\lambda
\end{array}\right] \text {. }
$$

Consider now the symmetric equilibrium where $p_{i}=p, x_{i}=x ; \frac{\partial \lambda}{\partial p_{i}}=\lambda_{i}$, $\frac{\partial f_{i}}{\partial x_{i}}=f_{i i}, \frac{\partial f_{i}}{\partial x_{j}}=f_{i j}, \frac{\partial x_{i}}{\partial p_{i}}=x_{i i}$ and $\frac{\partial x_{i}}{\partial p_{j}}=x_{i j}$ are all the same $(i \neq j)$. Assuming $p \lambda_{i}-f_{i j} \neq 0$, matrix $\mathbf{B}$ simplifies to:

$$
\mathbf{B}=\left[\begin{array}{ccc}
p \lambda_{i}-f_{i i} & \ldots & p \lambda_{i}-f_{i j} \\
\ldots & \ldots & \ldots \\
p \lambda_{i}-f_{i j} & \ldots & p \mu_{i}-f_{i i}
\end{array}\right]=\left(p \lambda_{i}-f_{i j}\right)\left[\iota \iota^{\prime}-\frac{f_{i i}-f_{i j}}{p \lambda_{i}-f_{i j}} \mathbf{I}\right]
$$

where $\iota$ is the relevant unit vector. Applying the Sherman-Morrison formula ${ }^{27}$ to invert it, we have:

$$
\begin{aligned}
\mathbf{B}^{-1} & =\frac{1}{p \lambda_{i}-f_{i j}}\left[-\frac{p \lambda_{i}-f_{i j}}{f_{i i}-f_{i j}} \mathbf{I}-\frac{\frac{p \lambda_{i}-f_{i j}}{f_{i i}-f_{i j}} \mathbf{I} \boldsymbol{\iota} \boldsymbol{\iota}^{\prime} \frac{p \lambda_{i}-f_{i j}}{f_{i i}-f_{i j}} \mathbf{I}}{1-\iota^{\prime} \frac{p \lambda_{i}-f_{i j}}{f_{i i}-f_{i j}} \mathbf{I} \iota}\right] \\
& =\frac{1}{p \lambda_{i}-f_{i j}}\left[-\frac{p \lambda_{i}-f_{i j}}{f_{i i}-f_{i j}} \mathbf{I}-\frac{\left[\frac{p \lambda_{i}-f_{i j}}{f_{i i}-f_{i j}}\right]^{2}}{1-n \frac{p \lambda_{i}-f_{i j}}{f_{i i}-f_{i j}}} \mathbf{1}\right] \\
& =-\frac{1}{f_{i i}-f_{i j}}\left[\mathbf{I}+\frac{p \lambda_{i}-f_{i j}}{f_{i i}-f_{i j}-n\left(p \lambda_{i}-f_{i j}\right)} \mathbf{1}\right]
\end{aligned}
$$

(which requires $0 \neq f_{i i}-f_{i j} \neq n\left(p \lambda_{i}-f_{i j}\right)$ ), where $\mathbf{1}$ is the relevant unit matrix. Since:

$$
D_{\mathbf{p}} \mathbf{x}(p \boldsymbol{\iota})=-\lambda \mathbf{B}^{-1}=\frac{\lambda}{f_{i i}-f_{i j}}\left[\mathbf{I}+\frac{p \lambda_{i}-f_{i j}}{f_{i i}-f_{i j}-n\left(p \lambda_{i}-f_{i j}\right)} \mathbf{1}\right],
$$

we get immediately:

$$
\frac{\partial x_{i}}{\partial p_{i}}=\frac{\lambda}{f_{i i}-f_{i j}}\left[1+\frac{p \lambda_{i}-f_{i j}}{f_{i i}-f_{i j}-n\left(p \lambda_{i}-f_{i j}\right)}\right],
$$

where $\lambda=n f_{i} x / E, p \lambda_{i}=\frac{E}{n x} \frac{(n-1) f_{i j} x+f_{i i} x+f_{i}}{E}=\frac{(n-1) f_{i j} x+f_{i i} x+f_{i}}{n x}, p \lambda_{i}-f_{i j}=$ $\frac{-f_{i j} x+f_{i i} x+f_{i}}{n x}, f_{i i}-f_{i j}-n\left(p \lambda_{i}-f_{i j}\right)=-\frac{f_{i}}{x}$. This proves the second equality in (13).

\footnotetext{
${ }^{27}$ The Sherman-Morrison formula says that:

$$
\left(\mathbf{A}+\mathbf{x y}^{\prime}\right)^{-1}=\mathbf{A}^{-1}-\frac{\mathbf{A}^{-1} \mathbf{x y}^{\prime} \mathbf{A}^{-1}}{1+\mathbf{y}^{\prime} \mathbf{A}^{-1} \mathbf{x}}
$$
}

if $\mathbf{A}$ is invertible and $1+\mathbf{y}^{\prime} \mathbf{A}^{-1} \mathbf{x} \neq \mathbf{0}$ : see Sherman and Morrison (1950). 
Let us consider the "dual approach", based on the direct Mashallian demand system $\mathbf{x}(\mathbf{s})$. The Bertrand equilibrium can be found by direct calculation. To find the Cournot equilibrium we must obtain the elasticity of the inverse demand from the inversion of the direct demand system, which in matrix notation is implicitly defined by $\mathbf{x} \mu(\mathbf{s})-\mathbf{g}(\mathbf{s})=0$, where we simplify notation with $\mu(\mathbf{s})=$ $\mu^{n}(\mathbf{s})$. By the Implicit Function Theorem we have:

$$
\mathbf{A} D_{\mathbf{x}} \mathbf{s}(\mathbf{x})=-\mu(\mathbf{s}) \mathbf{I},
$$

with

$$
\begin{gathered}
\mathbf{A}=\mathbf{x} D_{\mathbf{s}} \mu(\mathbf{s})^{\prime}-D_{s} \mathbf{g}(\mathbf{s})=\left[\begin{array}{ccc}
x_{1} \frac{\partial \mu}{\partial s_{1}}-\frac{\partial g_{1}}{\partial s_{1}} & \ldots & x_{1} \frac{\partial \mu}{\partial s_{n}}-\frac{\partial g_{1}}{\partial s_{n}} \\
\ldots & \ldots & \ldots \\
x_{n} \frac{\partial \mu}{\partial s_{1}}-\frac{\partial g_{n}}{\partial s_{1}} & \ldots & x_{n} \frac{\partial \mu}{\partial s_{n}}-\frac{\partial g_{n}}{\partial s_{n}}
\end{array}\right], \\
D_{\mathbf{x}} \mathbf{s}(\mathbf{x})=\left[\begin{array}{ccc}
\frac{\partial s_{1}}{\partial x_{1}} & \ldots & \frac{\partial s_{1}}{\partial x_{n}} \\
\ldots & \ldots & \ldots \\
\frac{\partial s_{n}}{\partial x_{1}} & \ldots & \frac{\partial s_{n}}{\partial x_{n}}
\end{array}\right] \text { and }-\mu(\mathbf{s}) \mathbf{I}=\left[\begin{array}{ccc}
-\mu & \ldots & 0 \\
\ldots & \ldots & \ldots \\
0 & \ldots & -\mu
\end{array}\right] .
\end{gathered}
$$

Consider now the symmetric equilibrium. Assuming $x \mu_{i}-g_{i j} \neq 0$ we get:

$$
\mathbf{A}=\left[\begin{array}{ccc}
x \mu_{i}-g_{i i} & \ldots & x \mu_{i}-g_{i j} \\
\ldots & \ldots & \ldots \\
x \mu_{i}-g_{i j} & \ldots & x \mu_{i}-g_{i i}
\end{array}\right]=\left(x \mu_{i}-g_{i j}\right)\left[\iota \iota^{\prime}-\frac{g_{i i}-g_{i j}}{x \mu_{i}-g_{i j}} \mathbf{I}\right] .
$$

Using the Sherman-Morrison formula we get:

$$
\mathbf{A}^{-1}=-\frac{1}{g_{i i}-g_{i j}}\left[\mathbf{I}+\frac{x \mu_{i}-g_{i j}}{g_{i i}-g_{i j}-n\left(x \mu_{i}-g_{i j}\right)} \mathbf{1}\right]
$$

which requires $g_{i i}-g_{i j} \neq 0$ and $g_{i i}-g_{i j} \neq n\left(x \mu_{i}-g_{i j}\right)$. Since:

$$
D_{\mathbf{x}} \mathbf{s}(\mathbf{x})=-\mu(\mathbf{s}) \mathbf{A}^{-1}=\frac{\mu(\mathbf{s})}{g_{i i}-g_{i j}}\left[\mathbf{I}+\frac{x \mu_{i}-g_{i j}}{g_{i i}-g_{i j}-n\left(x \mu_{i}-g_{i j}\right)} \mathbf{1}\right],
$$

we get immediately:

$$
\frac{\partial s_{i}(\mathbf{x})}{\partial x_{i}}=\frac{\mu(\mathbf{s})}{g_{i i}-g_{i j}}\left[1+\frac{x \mu_{i}-g_{i j}}{g_{i i}-g_{i j}-n\left(x \mu_{i}-g_{i j}\right)}\right],
$$

which delivers the second equality in (11).

\section{C: Generalized Leontief preferences}

The so-called Generalized Leontief (Diewert, 1971) homothetic expenditure function corresponding to (20) is given by:

$$
E(\mathbf{p}, U)=U \sum_{i=1}^{N} \sum_{j=1}^{N} b_{i j} \sqrt{p_{i} p_{j}}=U \sqrt{\mathbf{p}}^{\prime} \mathbf{B} \sqrt{\mathbf{p}}
$$


where to achieve symmetry it is assumed that $b_{i j}=b>0$ and $b_{i i}=\underline{b}, i, j=$ $1, \ldots, n, i \neq j$, and $N$ is the total number of conceivable varieties, to be kept constant. Without loss of generality, we can further normalize $b=1$. Then:

$$
\begin{aligned}
\mathbf{x}(\mathbf{p}, U) & =D_{\mathbf{p}} E(\mathbf{p}, U)=U(\widehat{\sqrt{\mathbf{p}}})^{-1} \mathbf{B} \sqrt{\mathbf{p}}, \\
x_{i}(\mathbf{p}, U) & =U \sum_{j=1}^{N} b_{i j} \sqrt{\frac{p_{j}}{p_{i}}}
\end{aligned}
$$

where $x_{i}(\mathbf{p}, U)$ is the compensated (Hicksian) demand of variety $i$ and the sym$\mathrm{bol}^{\wedge}$ denotes vector diagonalization. Notice that $\underline{b}$ does not enter the Jacobian $D_{\mathbf{p}} x(\mathbf{p}, U)$, and thus the regularity conditions it has to satisfy (negativesemidefiniteness). However, if all prices are the same $x_{i}(p \boldsymbol{\iota}, U)=U[\underline{b}+(N-1)]$, which suggests the condition $(N-1) b>-\underline{b}$.

Finally, the value of $\underline{b}$ may be crucial to account for some variety not being available for consumption, because not offered within the market. Notice that using (67) one can write (in matrix form):

$$
E(\mathbf{p}, U)=U \sqrt{\mathbf{p}}^{\prime} \mathbf{B} \sqrt{\mathbf{p}}=\mathbf{p}^{\prime} \mathbf{x},
$$

as it has to be by homogeneity and Shephard's Lemma. Now, let us partition the varieties into the set $I$ of varieties actually consumed, $i=1,2, \ldots, n$, and the set $J$ of varieties not available, $j=n+1, n+2, \ldots, N$, with $p^{I}=\left[p_{1}, \ldots p_{n}\right]$, $p^{J}=\left[p_{n+1, \ldots}, p_{N}\right], B=\left[\begin{array}{ll}\mathbf{B}^{I I} & \mathbf{B}^{I J} \\ \mathbf{B}^{J I} & \mathbf{B}^{J J}\end{array}\right]$, and similarly. By $(67)$ :

$$
\mathbf{x}^{J}=U\left(\widehat{\sqrt{\mathbf{p}^{J}}}\right)^{-1}\left[\begin{array}{ll}
\mathbf{B}^{J I} & \mathbf{B}^{J J}
\end{array}\right] \sqrt{\mathbf{p}}=[\mathbf{0}],
$$

requiring

$$
\sqrt{\mathbf{p}^{J}}=-\left(\mathbf{B}^{J J}\right)^{-1} \mathbf{B}^{J I} \sqrt{\mathbf{p}^{I}}
$$

if $B^{J J}$ is invertible. However, $\mathbf{B}^{J J}$ can be written as

$$
\mathbf{B}^{J J}=(1-\underline{b})\left[\frac{1}{1-\underline{b}} \mathbf{1}_{N-n, N-n}-\mathbf{I}_{N-n, N-n}\right]
$$

where $\mathbf{I}$ is the relevant identity matrix. Since $\mathbf{1}_{N-n, N-n}$ has eigenvalues $(N-n)$ and zero, $\mathbf{B}^{J J}$ has eigenvalues $(N-n-1)+\underline{b}$ and $\underline{b}-1$, and it will then be invertible if $(N-n-1) \neq-\underline{b} \neq-1$. Since

$$
\begin{aligned}
\mathbf{x}^{I} & =U\left(\widehat{\sqrt{\mathbf{p}^{I}}}\right)^{-1}\left[\begin{array}{ll}
\mathbf{B}^{I I} & \mathbf{B}^{I J}
\end{array}\right]\left[\begin{array}{c}
\sqrt{\mathbf{p}^{I}} \\
\sqrt{\mathbf{p}^{J}}
\end{array}\right] \\
& =U\left(\widehat{\sqrt{\mathbf{p}^{I}}}\right)^{-1}\left[\begin{array}{ll}
\mathbf{B}^{I I} & \mathbf{B}^{I J}
\end{array}\right]\left[\begin{array}{c}
\sqrt{\mathbf{p}^{I}} \\
-\left(\mathbf{B}^{J J}\right)^{-1} \mathbf{B}^{J I} \sqrt{\mathbf{p}^{I}}
\end{array}\right],
\end{aligned}
$$


we obtain

$$
\begin{gathered}
E^{n}\left(\mathbf{p}, U^{n}\right)=\mathbf{p}^{I \prime} \mathbf{x}^{I}=U^{n} \mathbf{p}^{I \prime}\left(\widehat{\sqrt{\mathbf{p}^{I}}}\right)^{-1}\left[\begin{array}{ll}
\mathbf{B}^{I I} & \mathbf{B}^{I J}
\end{array}\right]\left[\begin{array}{c}
\sqrt{\mathbf{p}^{I}} \\
-\left(\mathbf{B}^{J J}\right)^{-1} \mathbf{B}^{J I} \sqrt{\mathbf{p}^{I}}
\end{array}\right] \\
=U^{n}{\sqrt{\mathbf{p}^{I}}}^{\prime}\left[\begin{array}{ll}
\mathbf{B}^{I I} & \mathbf{B}^{I J}
\end{array}\right]\left[\begin{array}{c}
\sqrt{\mathbf{p}^{I}} \\
-\left(\mathbf{B}^{J J}\right)^{-1} \mathbf{B}^{J I} \sqrt{\mathbf{p}^{I}}
\end{array}\right] \\
=U^{n}\left[\begin{array}{l}
\sqrt{\mathbf{p}^{I}} \mathbf{B}^{I I} \sqrt{\mathbf{p}^{I}}-\sqrt{\mathbf{p}^{I}} \mathbf{B}^{I J}\left(\mathbf{B}^{J J}\right)^{-1} \mathbf{B}^{J I} \sqrt{\mathbf{p}^{I}}
\end{array}\right] \\
=U^{n}{\sqrt{\mathbf{p}^{I}}}^{\prime}\left[\mathbf{B}^{I I}-\mathbf{B}^{I J}\left(\mathbf{B}^{J J}\right)^{-1} \mathbf{B}^{J I}\right] \sqrt{\mathbf{p}^{I}} \\
=U^{n} \sqrt{\mathbf{p}^{I}} \mathbf{A} \sqrt{\mathbf{p}^{I}},
\end{gathered}
$$

where $\mathbf{A}=\left[\mathbf{B}^{I I}-\mathbf{B}^{I J}\left(\mathbf{B}^{J J}\right)^{-1} \mathbf{B}^{J I}\right]$ is a simmetric matrix and $n$ is the number of available varieties.

Notice that $\mathbf{B}^{I J}=\mathbf{B}^{J I^{\prime}}=\mathbf{1}_{n, N-n}$, where $\mathbf{1}_{n, N-n}$ is a $(n, N-n)$ matrix with unit entries, and $\mathbf{B}^{J J}$ is a $(N-n, N-n)$ square matrix whose diagonal elements are equal to $\underline{b}$ and extradiagonal elements are equal to 1 . Since:

$$
\mathbf{B}^{J J} \mathbf{1}_{N-n}=[(N-n)-1+\underline{b}] \mathbf{1}_{N-n}=\lambda \mathbf{1}_{N-n}
$$

has the obvious solution $\lambda=(N-n-1)+\underline{b}$, then $\mathbf{B}^{J J}$ has a unit eigenvector associated to the eigenvalue $N-n-1+\underline{b}$. Accordingly, its inverse has a unit eigenvector associated to an eigenvalue given by $[(N-n-1)+\underline{b}]^{-1}$. From this we can compute:

$$
\left(\mathbf{B}^{J J}\right)^{-1} \mathbf{B}^{J I}=\frac{1}{(N-n-1)+\underline{b}} \mathbf{1}_{N-n, n}
$$

and

$$
\mathbf{B}^{I J}\left(\mathbf{B}^{J J}\right)^{-1} \mathbf{B}^{J I}=\frac{N-n}{N-n-1+\underline{b}} \mathbf{1}_{n, n} .
$$

Since $\mathbf{B}^{I I}=\left[\mathbf{1}_{n, n}-(1-\underline{b}) \mathbf{I}_{n, n}\right]$, it follows that:

$$
\begin{aligned}
\mathbf{A} & =\mathbf{B}^{I I}-\mathbf{B}^{I J}\left(\mathbf{B}^{J J}\right)^{-1} \mathbf{B}^{J I}=\left[\mathbf{1}_{n, n}-(1-\underline{b}) \mathbf{I}_{n, n}\right]-\frac{N-n}{N-n-1+\underline{b}} \mathbf{1}_{n, n} \\
& =\frac{\underline{b}-1}{N-n-1+\underline{b}} \mathbf{1}_{n, n}-(1-\underline{b}) \mathbf{I}_{n, n} .
\end{aligned}
$$

Accordingly,

$$
a_{i i}=\underline{a}=\frac{(\underline{b}-1)[N-n+\underline{b}]}{N-n-1+\underline{b}}, \quad a_{i j}=a=\frac{\underline{b}-1}{N-n-1+\underline{b}} .
$$

Notice that the final normalization $\underline{b}=-(N-2)$ satisfies all our assumptions. In particular, $\underline{b}<0,(N-1)>-\underline{b}$ and $\mathbf{B}^{J J}$ is a negative definite matrix. 


\section{D: Generalized quadratic preferences}

Consider the following indirect utility function:

$$
V=\sum_{i=1}^{N} \sum_{j=1}^{N} \beta_{i j} s_{i} s_{j}-\sum_{j=1}^{N} \alpha_{j} s_{j}
$$

where to achieve symmetry it is assumed that $\alpha_{j}=\alpha, \beta_{i j}=\beta$ and $\beta_{i i}=\beta$, $i, j=1, \ldots, n, i \neq j$, and $N$ is always the total number of potential varieties. Accordingly, $V(\mathbf{s})=\mathbf{s}^{\prime} \boldsymbol{\beta} \mathbf{s}-\alpha \overline{\mathbf{s}}$, where $\overline{\mathbf{s}}=\sum_{j=1}^{N} s_{j}$. Notice that:

$$
\boldsymbol{\beta}=\beta \mathbf{1}_{N x N}-(\beta-\underline{\beta}) \mathbf{I}_{N x N}=(\beta-\underline{\beta})\left[\frac{\beta}{(\beta-\underline{\beta})} \mathbf{1}_{N x N}-\mathbf{I}_{N x N}\right]:
$$

since $\mathbf{1}_{N x N}$ has eigenvalues $N$ and 0 , the eigenvalues of $\boldsymbol{\beta}$ are then given by $(N-1) \beta+\underline{\beta}$ and $\underline{\beta}-\beta$. Since:

$$
\begin{aligned}
& D_{\mathbf{s}} V(\mathbf{s})=2 \beta \mathbf{s}-\alpha \boldsymbol{\iota} \quad D_{\mathbf{s}}^{2} V(\mathbf{s})=2 \boldsymbol{\beta} \\
& \frac{\partial V(s \boldsymbol{\iota})}{\partial s_{i}}=2 \sum_{j=1}^{N} \beta_{i j} s-\alpha=2 s[\underline{\beta}+(N-1) \beta]-\alpha,
\end{aligned}
$$

where $\iota=\mathbf{1}_{N}$, to satisfy the conditions of monotonicity and (quasi-)convexity of $V$ we assume that $\alpha>0$, and that $\boldsymbol{\beta}$ is a positive semidefinite matrix. In particular, we assume that $(N-1) \beta \geq-\underline{\beta}, \underline{\beta}>\beta, \underline{\beta}>0$ and restrict attention to the case in which $\alpha \boldsymbol{\iota}>2 \beta$ s. We obtain:

$$
\mathbf{x}(\mathbf{s})=\frac{D_{\mathbf{s}} V(\mathbf{s})}{D_{\mathbf{s}} V(\mathbf{s})^{\prime} \mathbf{s}}=-\frac{\alpha \iota-2 \beta \mathbf{s}}{2 \mathbf{s}^{\prime} \boldsymbol{\beta} \mathbf{s}-\alpha \iota^{\prime} \mathbf{s}}
$$

with

$$
V(s \iota)=N s\{s[(N-1) \beta+\underline{\beta}]-\alpha\}<0 .
$$

Let us partition the varieties into the set $I$ of varieties actually consumed, $i=1,2, \ldots, n$, and the set $J$ of varieties not available, $j=n+1, n+2, \ldots, N$, with $\mathbf{s}^{I}=\left[s_{1}, \ldots s_{n}\right], \mathbf{s}^{J}=\left[s_{n+1}, \ldots s_{N}\right], \boldsymbol{\beta}=\left[\begin{array}{ll}\boldsymbol{\beta}^{I I} & \boldsymbol{\beta}^{I J} \\ \boldsymbol{\beta}^{J I} & \boldsymbol{\beta}^{J J}\end{array}\right]$, and similarly. Notice that, by $(71), \mathbf{x}^{J}=[\mathbf{0}]$ requires $\alpha \iota=2\left[\begin{array}{ll}\boldsymbol{\beta}^{J I} & \boldsymbol{\beta}^{J J}\end{array}\right] \mathbf{s}=2\left(\boldsymbol{\beta}^{J I} \mathbf{s}^{I}+\boldsymbol{\beta}^{J J} \mathbf{s}^{J}\right)$, implying:

$$
\mathbf{s}^{J}=\frac{\alpha}{2}\left(\boldsymbol{\beta}^{J J}\right)^{-1} \iota-\left(\boldsymbol{\beta}^{J J}\right)^{-1} \boldsymbol{\beta}^{J I} \mathbf{s}^{I}
$$

since $\boldsymbol{\beta}^{J J}=(\beta-\underline{\beta})\left[\frac{\beta}{(\beta-\underline{\beta})} \mathbf{1}_{(N-n) x(N-n)}-\mathbf{I}_{(N-n) x(N-n)}\right]$ is invertible (and positive definite) under our assumptions. Moreover:

$$
\boldsymbol{\beta}^{J J} \boldsymbol{\iota}=(\beta-\underline{\beta})\left[\frac{\beta}{(\beta-\underline{\beta})}(N-n)-1\right] \boldsymbol{\iota},
$$


and thus $\boldsymbol{\beta}^{J J}$ has a unit eigenvector associated to the positive eigenvalue $\beta(N-n-1)+$ $\underline{\beta}$. This implies that $\left(\boldsymbol{\beta}^{J J}\right)^{-1}$ has a unit eigenvector associated to the positive eigenvalue $1 /[\beta(N-n-1)+\underline{\beta}]$. Since $\boldsymbol{\beta}^{J I}=\beta \mathbf{1}_{(N-n) x n}$ we get:

$$
\mathbf{s}^{J}=\left[\frac{\alpha}{2}-\beta \overline{\mathbf{s}^{I}}\right]\left(\boldsymbol{\beta}^{J J}\right)^{-1} \iota=\frac{\frac{\alpha}{2}-\beta \overline{\mathbf{s}^{I}}}{\beta(N-n-1)+\underline{\beta}} \iota>0 .
$$

which identifies the choke-off (normalized) prices of the $N-n$ unproduced varieties as functions of the (normalized) prices of the $n$ varieties actually offered in market. Using (72) yields:

$$
\begin{aligned}
& V^{n}(\mathbf{s})= \mathbf{s}^{\prime} \boldsymbol{\beta} \mathbf{s}-\alpha \iota^{\prime} \mathbf{s}=\left[\begin{array}{ll}
\mathbf{s}^{I \prime} & \mathbf{s}^{J \prime}
\end{array}\right]\left[\begin{array}{ll}
\boldsymbol{\beta}^{I I} & \boldsymbol{\beta}^{I J} \\
\boldsymbol{\beta}^{J I} & \boldsymbol{\beta}^{J J}
\end{array}\right]\left[\begin{array}{l}
\mathbf{s}^{I} \\
\mathbf{s}^{J}
\end{array}\right]-\alpha \boldsymbol{\iota}^{\prime}\left[\begin{array}{l}
\mathbf{s}^{I} \\
\mathbf{s}^{j}
\end{array}\right] \\
&= {\left[\begin{array}{ll}
\mathbf{s}^{I \prime} & \mathbf{s}^{J \prime}
\end{array}\right]\left[\begin{array}{c}
\boldsymbol{\beta}^{I I} \mathbf{s}^{I}+\boldsymbol{\beta}^{I J} \mathbf{s}^{J} \\
\boldsymbol{\beta}^{J I} \mathbf{s}^{I}+\boldsymbol{\beta}^{J J} \mathbf{s}^{J}
\end{array}\right]-\alpha \boldsymbol{\iota}^{\prime}\left[\begin{array}{c}
\mathbf{s}^{I} \\
\mathbf{s}^{j}
\end{array}\right] } \\
&= \mathbf{s}^{I \prime} \boldsymbol{\beta}^{I I} \mathbf{s}^{I}+\mathbf{s}^{I \prime} \boldsymbol{\beta}^{I J} \mathbf{s}^{J}+\mathbf{s}^{J \prime} \boldsymbol{\beta}^{J I} \mathbf{s}^{I}+\mathbf{s}^{J \prime} \boldsymbol{\beta}^{J J} \mathbf{s}^{J}-\alpha \overline{\mathbf{s}^{I}}-\alpha \overline{\mathbf{s}^{J}} \\
&= \mathbf{s}^{I \prime} \boldsymbol{\beta}^{I I} \mathbf{s}^{I}-\alpha \overline{\mathbf{s}^{I}}+2(N-n) \beta \overline{\mathbf{s}^{I}} \frac{\frac{\alpha}{2}-\beta \overline{\mathbf{s}^{I}}}{\beta(N-n-1)+\underline{\beta}} \\
&+(N-n) \frac{\left(\frac{\alpha}{2}-\beta \overline{\mathbf{s}^{I}}\right)^{2}}{\beta(N-n-1)+\underline{\beta}}-\alpha(N-n) \frac{\frac{\alpha}{2}-\beta(N-n-1)+\underline{\beta}}{(n-N)\left(\frac{\alpha}{2}-\beta \overline{\mathbf{s}^{I}}\right)^{2}} \\
&=\mathbf{s}^{I \prime} \boldsymbol{\beta}^{I I} \mathbf{s}^{I}-\alpha \overline{\mathbf{s}^{I}}+\frac{(n-n-1)+\underline{\beta}}{\beta(N-n-1)}
\end{aligned}
$$

or:

$$
V^{n}(\mathbf{s})=\mathbf{s}^{I \prime} \boldsymbol{\beta}^{I I} \mathbf{s}^{I}+\frac{(n-N)\left(\frac{\alpha^{2}}{4}+\beta^{2} \overline{\mathbf{s}}^{2}\right)-\alpha \overline{\mathbf{s}^{I}}(\underline{\beta}-\beta)}{\beta(N-n-1)+\underline{\beta}} .
$$

Let us normalize $\beta=-b<0, \beta=b(N-1)$. Then all our assumptions are satisfied and we reach the indirect utility:

$$
V^{n}(\mathbf{s})=\sum_{i=1}^{n} \sum_{j=1}^{n} \beta_{i j} s_{i} s_{j}+\frac{N-n}{n \beta} \frac{\alpha^{2}}{4}+\frac{N-n}{n} \beta{\overline{\mathbf{s}^{I}}}^{2}-\frac{\alpha N \overline{\mathbf{s}^{I}}}{n} .
$$

which endogenously depends on the number of provided varieties and their prices. 